$\mathrm{DCPT} / 08 / 172$

$\operatorname{IPPP} / 08 / 86$

BONN-TH-2008-17

\title{
HiggsBounds: Confronting Arbitrary Higgs Sectors with Exclusion Bounds from LEP and the Tevatron
}

\author{
P. Bechtle ${ }^{\mathrm{a}}$, O. Brein ${ }^{\mathrm{b}}$, S. Heinemeyer ${ }^{\mathrm{c}}$, G. Weiglein ${ }^{\mathrm{b}}$ \\ and K.E. Williams ${ }^{\mathrm{d}}$. \\ ${ }^{a}$ DESY, Notkestrasse 85, 22607 Hamburg, Germany \\ ${ }^{\mathrm{b}}$ Institute for Particle Physics Phenomenology, Durham University, \\ Durham, DH1 3LE, United Kingdom \\ ${ }^{\mathrm{c}}$ Instituto de Fisica de Cantabria (CSIC-UC), Santander, Spain \\ ${ }^{\mathrm{d}}$ Bethe Center for Theoretical Physics, Physikalisches Institut der Universität \\ Bonn, Nussallee 12, 53115 Bonn, Germany
}

\begin{abstract}
HiggsBounds is a computer code that tests theoretical predictions of models with arbitrary Higgs sectors against the exclusion bounds obtained from the Higgs searches at LEP and the Tevatron. The included experimental information comprises exclusion bounds at $95 \%$ C.L. on topological cross sections. In order to determine which search topology has the highest exclusion power, the program also includes, for each topology, information from the experiments on the expected exclusion bound, which would have been observed in case of a pure background distribution. Using the predictions of the desired model provided by the user as input, HiggsBounds determines the most sensitive channel and tests whether the considered parameter point is excluded at the $95 \%$ C.L. HiggsBounds is available as a Fortran 77 and Fortran 90 code. The code can be invoked as a command line version, a subroutine version and an online version. Examples of exclusion bounds obtained with HiggsBounds are discussed for the Standard Model, for a model with a fourth generation of quarks and leptons and for the Minimal Supersymmetric Standard Model with and without $\mathcal{C P}$-violation. The experimental information on the exclusion bounds currently implemented in HiggsBounds will be updated as new results from the Higgs searches become available.
\end{abstract}

Key words: Higgs bosons, Higgs search, LEP, Tevatron, Beyond the Standard Model 


\section{PROGRAM SUMMARY}

Manuscript Title: HiggsBounds: Confronting Arbitrary Higgs Sectors with Exclusion Bounds from LEP and the Tevatron

Authors: P. Bechtle, O. Brein, S. Heinemeyer, G. Weiglein, K.E. Williams

Program Title: HiggsBounds

Journal Reference:

Catalogue identifier:

Licensing provisions:

Program requirements: HiggsBounds can be built with any compatible Fortran 77 or Fortran 90 compiler. The program has been tested on x86 CPUs running under Linux (Ubuntu 8.04) and with the following compilers: The Portland Group Inc. Fortran compilers (pgf77, pgf90), the GNU project Fortran compilers (g77, gfortran).

Programming language: Fortran 77, Fortran 90 (two code versions are offered)

RAM: minimum of about 6000 kbytes (dependent on the code version)

Keywords: Elementary Particle Physics; General, High Energy Physics and Computing; Higgs bosons; Higgs search; LEP; Tevatron; Beyond the Standard Model.

PACS: 14.80.Bn; 14.80.Cp; 12.60.Fr.

Classification:

External routines/libraries: HiggsBounds requires no external routines/libraries. Some sample programs in the distribution require the programs FeynHiggs 2.6.x or CPsuperH2 to be installed.

No. of lines in distributed program, including test data, etc.: 61645

No. of bytes in distributed program, including test data, etc.: 13721912

Distribution format: tar.gz

Nature of problem: Determine whether a parameter point of a given model is excluded or allowed by LEP and Tevatron Higgs-boson search results.

Solution method: The most sensitive channel from LEP and Tevatron searches is determined and subsequently applied to test this parameter point. The test requires as input model predictions for the Higgs-boson masses, branching ratios and ratios of production cross sections with respect to reference values.

Restrictions: In the current version, results from decay-mode independent Higgs searches and results of searches for charged Higgs bosons are not taken into account.

Running time: about 0.01 seconds (or less) for one parameter point using one processor of an Intel Core 2 Quad Q6600 CPU at 2.40GHz for sample model scenarios with three Higgs bosons. It depends on the complexity of the Higgs sector (e.g. the number of Higgs bosons and the number of open decay channels) and on the code version. 


\section{LONG WRITE-UP}

\section{Introduction}

A major goal of the particle physics programme at the high energy frontier, currently being pursued at the Fermilab Tevatron collider and soon to be taken up by the CERN Large Hadron Collider (LHC), is to unravel the nature of electroweak symmetry breaking. While the existence of the massive electroweak gauge bosons $\left(W^{ \pm}, Z\right)$, together with the successful description of their behaviour by non-abelian gauge theory, requires some form of electroweak symmetry breaking to be present in nature, the underlying dynamics is not known yet. An appealing theoretical suggestion for such dynamics is the Higgs mechanism [1], which implies the existence of one or more Higgs bosons (depending on the specific model considered). Therefore, the search for Higgs bosons is a major cornerstone in the physics programmes of past, present and future high energy colliders.

Many theoretical models employing the Higgs mechanism in order to account for electroweak symmetry breaking have been studied in the literature, of which the most popular ones are the Standard Model (SM) [2] and the Minimal Supersymmetric Standard Model (MSSM) [3]. Within the SM, the Higgs boson is the last undiscovered particle, whereas the MSSM has a richer Higgs sector, containing three neutral and two charged Higgs bosons. Among alternative theoretical models beyond the SM which lead to the prediction of Higgs bosons, the most prominent are the Two Higgs Doublet Model (THDM) [4, non-minimal supersymmetric extensions of the SM (e.g. extensions of the MSSM by an extra singlet superfield [5]), little Higgs models [6] and models with more than three spatial dimensions [7]. Furthermore, several models have been proposed which mainly extend the Higgs sector of the SM, e.g. extensions of the SM by electroweak singlet scalar fields [8], or the Private Higgs Model [9] which introduces one Higgs doublet for each fermion generation, or models with Higgs-dependent Yukawa couplings [10].

LEP has searched for the SM Higgs boson [11, the MSSM Higgs bosons [12], and other, more "exotic", manifestations of the Higgs mechanism [13]. After the termination of LEP, the search has been taken up again by the Tevatron. So far, no signals of Higgs bosons have been found, and LEP and Tevatron turned the non-observation of Higgs signals into cross section constraints. The constraints are provided by experiments in the form of limits on cross sections of individual signal topologies (such as $e^{+} e^{-} \rightarrow h_{i} Z \rightarrow b \bar{b} Z$ or $p \bar{p} \rightarrow h_{i} Z \rightarrow$ $b \bar{b} l^{+} l^{-}$) or in the form of combined limits for a specific model, such as the SM. In the latter case, the individual topological cross sections have been combined using the proportions of the individual contributions as predicted 
by the model.

An important part of the phenomenological exploration of physics models beyond the SM is the confrontation of such models with existing experimental constraints. In particular, checking the predictions of the model's Higgs sector with bounds from experimental searches for a Higgs boson is vital. In order to test whether the parameter space of a certain model is compatible with the limits from the Higgs searches at LEP and the Tevatron, it is, in general, not possible to employ the experimental results that have been obtained for the Higgs search in the SM (or any other specific model). This is because, in a general model of new physics, individual Higgs signal topologies will contribute in different proportions than, for instance, in the SM. Thus, for models for which no explicit experimental analysis has been carried out, or, like in the case of the MSSM, for model parameters that differ from the benchmark values chosen in the experimental analyses, one needs to resort to the limits on the individual topological cross sections provided by the experimental collaborations. These limits exist for various search channels from LEP and the Tevatron (the latter ones are frequently updated as new data becomes available). Comparing the predictions of a particular model with the existing experimental bounds on the various search topologies can be quite a tedious task as it involves the implementation of experimental results that are distributed over many different publications and combining these results requires a procedure to ensure the correct statistical interpretation of the exclusion bounds obtained on the parameter space of the model.

We present here the program HiggsBounds, which is a tool designed to facilitate the above task so that wide classes of models can easily be checked against the state-of-the-art results from Higgs searches. This should be useful for applications in Higgs phenomenology and model building. HiggsBounds takes theoretical Higgs sector predictions, e.g. for a particular parameter scenario of a model beyond the SM, as input and determines which Higgs search analysis has the highest exclusion power according to a list of expected exclusion limits from LEP and the Tevatron (an expected exclusion limit corresponds to the bound that one would obtain in the hypothetical case of an observed distribution that agrees precisely with the background expectation). In order to ensure the correct statistical interpretation of the obtained exclusion bound as a $95 \%$ C.L., the comparison of the model with the experimental limits has to be restricted to the single channel that possesses the highest statistical sensitivity for setting an exclusion limit. For this channel, the program then compares the theoretical prediction for the Higgs production cross section times decay branching ratio with the actual experimental limit and determines whether or not the considered parameter point of the model is excluded at 95\% C.L. So far, no bounds on charged Higgs bosons are taken into account. This will be included in a later version. HiggsBounds is model-independent, in that it can be used for models with arbitrary Higgs sectors. The input needed is the 
number of neutral Higgs bosons in the model and masses, decay branching ratios and ratios of production cross sections versus corresponding reference (usually SM) values for all neutral Higgs bosons in the model. The code has both a Fortran 77 and Fortran 90 version. It can be operated in a command line mode that can process input files in a variety of formats, as a subroutine suitable for inclusion in user applications, and as an online version, available at the URL: http://www.ippp.dur.ac.uk/HiggsBounds. HiggsBounds includes sample programs which demonstrate its usage. Sample programs which demonstrate how HiggsBounds can be used in conjunction with the widely used programs FeynHiggs [14,15, 16,17] and CPsuperH [18] for Higgs-sector predictions in the MSSM are provided by default.

The rest of the paper is organised as follows. In Section 2 we describe our general approach for the implementation of experimental Higgs constraints followed by specific details on the implementation of LEP and Tevatron results. In Section 3, the general operating instructions for HiggsBounds are specified and accompanied by small sample programs, while in Section 4 some examples of use for HiggsBounds are described. Section 5 contains a summary and outlook and is followed by an appendix which contains details on the definition and statistical interpretation of the confidence limits.

\section{Implementation of experimental Higgs constraints}

\subsection{General approach}

Tevatron and LEP turn(ed) the non-observation of Higgs signals into 95\% C.L. upper limits on cross sections for individual signal topologies. These limits are given as functions of the anticipated mass of the Higgs boson. By a certain Higgs signal topology $X$, we mean a specific combination of a single (double) Higgs boson production process, $P(h)\left(P\left(h_{1}, h_{2}\right)\right)$, and decay final state(s) $F$ of the Higgs boson(s) $h\left(h_{1}, h_{2}\right)$. In the limit of a narrow-width Higgs boson the cross section $\sigma(X)$ of the signal topology $X$ factorises into the on-shell Higgs production cross sections $\sigma(P)$ times the appropriate Higgs decay branching $\operatorname{ratio}(\mathrm{s}) \operatorname{BR}(h \rightarrow F)\left(\mathrm{BR}\left(h_{1}, h_{2} \rightarrow F\right)\right)$.

The Tevatron and LEP cross section limits are understood to be applicable to models which do not change the signature of the background processes considerably 1 which do not significantly change the kinematical distributions of

$\overline{1}$ This is not such a strong extra restriction on new physics models as it may seem, because viable models are required to agree with present experimental results which are well described by the SM, such as the LEP and SLD EW precision data [19] and 
the signal topology $X$ (e.g. $\eta, p_{T}$ distributions of the final state particles) from what has been assumed in the corresponding analysis. This is an unavoidable limitation of the standard approach adopted in the literature of expressing search results in terms of limits on total cross sections times branching ratios for certain signal topologies. Detection efficiencies, for instance the b-jet tagging efficiency, usually depend on the kinematical configuration of the signal event (particularly the $\eta$ and $p_{T}$ of the final state particles). Therefore, a dedicated experimental analysis for a model giving rise to kinematical distributions that strongly differ from the ones assumed in the analysis on which the published cross section limit is based would lead to a somewhat modified cross section limit. For instance, applying the cross section limit of a Tevatron analysis which relies on b-jet tagging to a model where the distribution of the signal b-jets differs strongly from the one assumed in the Tevatron analysis would lead to a slightly too weak/strong limit if a higher/lower fraction of the signal b-jets than assumed in the analysis is radiated into the central part of the detector where the b-jet tagging efficiency is higher than in regions closer in angle to the beam axis. HiggsBounds provides three options for passing model predictions to the program: (hadr, part and effC). The extent to which the kinematical distributions may vary from those assumed in the analyses depends on which of these input options is chosen. We will discuss this issue in further detail in the sections describing the input options.

The Tevatron and LEP cross section limits are usually given for a narrowwidth Higgs boson. From the narrow-width limits, some experimental analyses extract limits for Higgs bosons with a substantial decay width by representing the signal of a "large-width" Higgs boson as a weighted sum of mock narrowwidth Higgs signals. Examples of this procedure can be found in [21,22,23|24]. In particular, 24 shows that the effect of a non-vanishing width on the extracted limit can be parametrised by a function of the Higgs mass $m_{h}$ and the ratio $\Gamma_{\text {tot }}(h) / m_{h}$. If model-independent information of this kind also became available (for a fine grid of points) for other analyses, this would greatly facilitate the task to implement width-dependent Higgs exclusion limits.

At present, we constrain the implementation of HiggsBounds to the case of narrow-width Higgs boson exclusion limits. Neglecting the width of a Higgs boson usually leads to tighter bounds on the topological cross sections than those obtained through taking the width into account. The user should be aware of this fact when considering exclusion bounds on parameter regions that give rise to very large Higgs-boson widths. In a future version of HiggsBounds we

the precision measurements of gauge boson production at LEP and the Tevatron [20]. These analyses are much more sensitive to changes in the background to Higgs searches than the Higgs searches themselves. Therefore, the models with new physics in the Higgs sector which have been considered in the literature usually do not show strong deviations from the SM in the background processes. 
plan to incorporate a proper width treatment for cases where width-dependent results are provided by the experimental collaborations. As mentioned above, HiggsBounds is not meant to be applied to models for which the background rates to the considered signal processes differ drastically to the SM case.

For some specific models (the SM and some benchmark scenarios of the MSSM) the results of several Higgs signal topologies have been combined by the experimental collaborations according to the relative contributions to the total cross section. In the combination, a detailed knowledge of the overlap between the individual experimental searches is used, which is not available using the limits on topological cross sections only. Hence, a combined limit, for instance for the SM, is more sensitive than a combination of individual topological limits. The use of detailed SM dependent information during the calculation of the combined limit prohibits its application to those Higgs bosons of new physics models which are not predicted to have all relevant couplings proportional to the SM values. However, such models can still contain one (or some) Higgs boson(s) which are SM-like. For those Higgs bosons, the SM combined limit can be applied.

The basic flow of HiggsBounds is as follows.

(1) The user provides (in a form to be specified below) for all neutral Higgs bosons $h_{i}\left(i=1, \ldots, n_{\text {Higgs }}\right)$ of the considered model the predictions for: Higgs masses, total decay widths, the relevant branching ratios for the decay of Higgs bosons $h_{i}$ into known ordinary particles (OP, i.e. SM fermions and gauge bosons) and into pairs of other Higgs bosons $h_{j}$, and the relevant cross sections for Higgs production processes $P$ normalised to reference (usually SM) values:

$$
\begin{aligned}
& m_{h_{i}}, \Gamma_{\text {tot }}\left(h_{i}\right), \operatorname{BR}\left(h_{i} \rightarrow \mathrm{OP}\right), \operatorname{BR}\left(h_{i} \rightarrow h_{j} h_{j}\right), \\
& \left.\frac{\sigma_{\text {model }}\left(P\left(h_{i}\right)\right)}{\sigma_{\text {ref }}\left(P(H), m_{H}\right)}\right|_{m_{H}=m_{h_{i}}},\left.\frac{\sigma_{\text {model }}\left(P\left(h_{i}, h_{j}\right)\right)}{\sigma_{\text {ref }}\left(P\left(H, H^{\prime}\right), m_{H}, m_{H^{\prime}}\right)}\right|_{m_{H, H^{\prime}}=m_{h_{i, j}}} .
\end{aligned}
$$

The precise definitions of the reference cross sections are given in the LEPand Tevatron-specific subsections below. The user can choose to specify all the above quantities directly, or, in the simplest form, one just needs to provide $m_{h_{i}}, \Gamma_{\text {tot }}\left(h_{i}\right), \operatorname{BR}\left(h_{i} \rightarrow h_{j} h_{j}\right)$ and effective couplings of the Higgs bosons to ordinary particles. The remaining branching ratios and cross section ratios listed above can then be calculated from this input in the effective coupling approximation. For the preparation of normalised input, the user may apply the SM predictions for Higgs boson production cross sections and decay branching ratios which HiggsBounds provides as external routines (see Table 12 in Section 3.3).

(2) With the above model input, the program calculates the model predictions for the cross section (normalised to a reference value in some cases) 
for each Higgs signal topology $Q_{\text {model }}(X)$. Depending on whether the exclusion result for a particular search topology has been given by the experimental collaborations as a relative or absolute limit, the program evaluates

$$
Q_{\text {model }}=\frac{[\sigma \times \mathrm{BR}]_{\text {model }}}{[\sigma \times \mathrm{BR}]_{\mathrm{ref}}} \text { or }[\sigma \times \mathrm{BR}]_{\text {model }}
$$

where $\sigma$ and BR denote the production process and decay branching ratio of the Higgs boson, respectively.

(3) From the experimental results, we read off the values $Q_{\text {obs }}(X)$ and $Q_{\text {expec }}(X)$ corresponding to the observed and expected $95 \%$ C.L. exclusion bound on the quantity $Q(X)$, respectively. To that end, we have implemented many of the available observed and expected exclusion limits (preliminary and final results) from LEP and the Tevatron as data tables which are read in by the program during start-up. In order to provide values for $Q_{\text {obs }}(X)$ and $Q_{\text {expec }}(X)$ for continuous Higgs mass values, the program interpolates $Q$-values linearly between neighbouring Higgs mass points given by the experimental collaborations. Details on the definition of $95 \%$ C.L. limits can be found in Appendix $\mathrm{A}$.

(4) The program determines the search topology $X_{0}$ with the highest ratio of $Q_{\text {model }}$ to expected $Q_{\text {expec}}$, i.e. out of all topologies $X$, it finds the channel $X_{0}$ where

$$
\frac{Q_{\text {model }}(X)}{Q_{\text {expec }}(X)}
$$

is maximal. This topology has the highest statistical sensitivity for excluding the model prediction.

(5) We then look at the ratio of $Q_{\text {model }}$ to the observed $Q_{\text {obs }}$ value for this channel $X_{0}$ and, if

$$
\frac{Q_{\text {model }}\left(X_{0}\right)}{Q_{\text {obs }}\left(X_{0}\right)}>1
$$

this particular parameter point is excluded by the corresponding experimental analysis for the search channel $X_{0}$ at $95 \%$ C.L. In order to ensure the correct statistical interpretation of the obtained exclusion bound as a 95\% C.L., it is crucial to perform the test of Eq. (2) only for exactly one search channel (namely the one with the highest statistical sensitivity), and not for all available search channels. In the latter case the derived constraint would in general not correspond to a constraint at $95 \%$ C.L., due to the maximally $5 \%$ probability of each individual comparison of $Q_{\text {model }}$ and $Q_{\text {obs }}$ to yield a false exclusion.

As additional information, the web version of the program HiggsBounds also informs the user which topologies had the second and third highest statistical sensitivity and whether or not the ratio of $Q_{\text {model }}$ to observed $95 \%$ C.L. limit 
$Q_{\text {obs }}$ was greater than one for those topologies. However, this information does not contribute to the decision about whether or not this parameter point is excluded.

There are a few more general implementation details which should be mentioned here.

Using SM combined limits, SM-likeness

In order to use the best available exclusion limit, it makes sense to use the results of the SM combined analyses for any Higgs boson predicted to behave in a similar way to the SM Higgs boson (in terms of its production and decay). A SM analysis, combining several signal topologies assumes that the relative contribution to the event rate by each included search topology is according to the proportions in the SM. For a given Higgs mass $m_{H}$, the only free parameter left in such analyses is one overall scale factor, which multiplies all Higgs signal cross sections and which is varied in order to determine the value for which 95\% C.L. exclusion occurs.

Therefore, the results of a SM analysis, combining $M$ signal topologies, are exactly applicable to a Higgs boson $h$ of an alternative model if for all of the $M$ search topologies $X_{n}$ the ratio of topological cross sections (model versus $\mathrm{SM})$ is independent of $X_{n}$, i.e.

$$
\frac{\sigma_{\text {model }}\left(X_{n}\right)}{\sigma_{\mathrm{SM}}\left(X_{n}\right)}=\text { const. }=: \alpha, \quad n=1, \ldots, M,
$$

with a proportionality constant $\alpha$, or equivalently

$$
\frac{\sigma_{\text {model }}\left(X_{n}\right)}{\sigma_{\text {model }}\left(X_{k}\right)}=\frac{\sigma_{\mathrm{SM}}\left(X_{n}\right)}{\sigma_{\mathrm{SM}}\left(X_{k}\right)} \quad \text { for all } n, k \in\{1, \ldots, M\} .
$$

For a model which passes the conditions (3) or (4) to sufficient accuracy for a set of $M$ Higgs search topologies, the cross section prediction for a combination of those topologies, normalised to the SM reference value, fulfils

$$
Q_{\text {model }}\left(\left\{X_{1}, \ldots X_{M}\right\}\right):=\frac{\sum_{n=1}^{M} \sigma_{\text {model }}\left(X_{n}\right)}{\sum_{n=1}^{M} \sigma_{\mathrm{SM}}\left(X_{n}\right)}=\alpha .
$$

Here and in the following, it is always understood that the mass of the SM Higgs boson $m_{H}$ is set to $m_{h}$. In the narrow-width approximation, which we assume to hold throughout (see the discussion above), each cross section $\sigma\left(X_{n}\right)$ of the Higgs signal topologies $X_{n}$ factorises into a production cross section $\sigma\left(P_{i}(h)\right)$ and decay branching ratio $\mathrm{BR}\left(h \rightarrow F_{k}\right)$, where $i$ and $k$ are functions of $n$, and $n=1, \ldots, M$. Note that the index $n$ labels distinct signal 
topologies, i.e. specific combinations of one Higgs production process with one decay final state. SM-likeness is fulfilled if

$$
\frac{\sigma\left(P_{i}(h)\right)_{\text {model }}}{\sigma\left(P_{i}(H)\right)_{\mathrm{SM}}} \frac{\mathrm{BR}_{\text {model }}\left(h \rightarrow F_{k}\right)}{\mathrm{BR}_{\mathrm{SM}}\left(H \rightarrow F_{k}\right)}=\text { const. }
$$

holds to sufficient accuracy for all $M$ topologies. If this is the case for a particular Higgs boson of a certain model, a SM analysis combining precisely those $M$ channels also applies to that Higgs boson.

In order to decide whether or not a Higgs boson of a certain model is sufficiently SM-like such that the corresponding SM combined analysis can be applied, we use in HiggsBounds a pragmatic definition of SM-likeness. For each of the $N_{\mathrm{CS}}$ distinct production cross sections $\sigma_{\text {model }}\left(P_{i}(h)\right)$ and the $N_{\mathrm{BR}}$ distinct decay branching ratios $\operatorname{BR}\left(h \rightarrow F_{k}\right)$ which appear in the list of $M$ signal topologies, we determine the normalised mean value and the deviation from the mean:

$$
\begin{aligned}
\bar{s} & =\frac{1}{N_{\mathrm{CS}}} \sum_{i=1}^{N_{\mathrm{CS}}} s_{i}, & \bar{b} & =\frac{1}{N_{\mathrm{BR}}} \sum_{k=1}^{N_{\mathrm{BR}}} b_{k}, \\
\delta s_{i} & =s_{i}-\bar{s}, & \delta b_{k} & =b_{k}-\bar{b}, \\
\text { with } s_{i} & =\frac{\sigma_{\text {model }}\left(P_{i}(h)\right)}{\sigma_{\mathrm{SM}}\left(P_{i}(H)\right)}, & b_{k} & =\frac{\mathrm{BR}_{\text {model }}\left(h \rightarrow F_{k}\right)}{\mathrm{BR}_{\mathrm{SM}}\left(H \rightarrow F_{k}\right)} .
\end{aligned}
$$

The approximate SM-likeness of the given model is then ensured if the deviations from a constant expression according to Eq. (6) are small. For a given combination $(i, k)$ of a production and decay process, the left hand side of Eq. (6) can be expressed as:

$$
\frac{\sigma_{\text {model }}\left(P_{i}(h)\right)}{\sigma_{\mathrm{SM}}\left(P_{i}(H)\right)} \frac{\mathrm{BR}_{\text {model }}\left(h \rightarrow F_{k}\right)}{\mathrm{BR}_{\mathrm{SM}}\left(H \rightarrow F_{k}\right)}=s_{i} b_{k}=\bar{s} \bar{b}\left(1+\frac{\delta s_{i}}{\bar{s}}+\frac{\delta b_{k}}{\bar{b}}+\frac{\delta s_{i} \delta b_{k}}{\bar{s} \bar{b}}\right) .
$$

In the program, we consider a corresponding SM analysis applicable if the maximum relative deviation from the mean value $\bar{s} \bar{b}$ satisfies

$$
\max _{i, k}\left(\frac{\delta s_{i}}{\bar{s}}+\frac{\delta b_{k}}{\bar{b}}+\frac{\delta s_{i} \delta b_{k}}{\bar{s} \bar{b}}\right)<\epsilon
$$

where we have chosen $\epsilon=2 \%$, i.e. the predictions for the different topological cross sections, normalised to the SM values, are required to be equal to each other with at least $2 \%$ accuracy. This restriction is quite stringent and may cause a SM combined analysis to be not applied to some models where it may actually be justifiable. The user can remedy this situation by changing the setting of the corresponding variable eps in the internal subroutine check_SM_likeness (Fortran 77) or SMlikeness (Fortran 90) in the code. 
So far, HiggsBounds uses analyses with SM combinations of topologies only from the Tevatron experiments. This is the most important case, as CDF and $\mathrm{D} \varnothing$ have not done combinations of their results for individual search topologies so far, while the LEP results we are using represent the combination of results from all four LEP experiments for each individual channel.

A more elaborate criterion for determining whether SM combined analyses are applicable to Higgs bosons of models beyond the SM can be sketched as follows. The variation of the ratios of signal topology cross sections (model versus SM) due to non-exact SM-likeness of the model prediction should stay within a fraction of the $1 \sigma$ band of the sensitivity of the observed limit of the analysis with respect to signal-like fluctuations. The fixed maximum deviation of $2 \%$, described above, lies well within the typical expected statistical fluctuation of the limits in the SM combined analyses. We plan to implement such a more elaborate criterion in future updates of the program.

\section{Adding of signal channels}

In models with more than one Higgs boson it can happen that some of the Higgs bosons, $h_{i}$, have masses which are quite close to each other, say

$$
\left|m_{h_{i}}-m_{h_{j}}\right| \leq \Delta m_{h}
$$

for certain $i$ and $j$. This is the case for instance in the parameter space of the $\left(\mathcal{C P}\right.$-conserving) MSSM for the heavy Higgs bosons $H^{0}$ and $A^{0}$ for $M_{A} \gg M_{Z}$. If the masses are so close to each other that their signals appear within a certain mass window, it may happen that the two signals are not experimentally distinguishable anymore, or, at least, that it makes sense to combine their signal contribution into one signal to be compared with the data.

In the narrow-width approximation (which is used in HiggsBounds, see the discussion above) it is assumed that the total widths of the Higgs bosons $\Gamma_{\text {tot }}\left(h_{i}\right)$ are all (much) smaller than their mutual mass differences,

$$
\Gamma_{\text {tot }}\left(h_{i}\right) \ll\left|m_{h_{i}}-m_{h_{k}}\right| \quad \text { for all } i \text { and } k \neq i
$$

In this case the signal channels factorise into production cross section and decay branching ratio, so that the addition of different signal cross sections may formally always be carried out where it is necessary. In parameter regions where the narrow-width approximation does not hold, however, one may worry about the possible effects of interference terms arising from squaring the amplitude of the full process involving both production and decay. It should be noted that even in cases where the total widths of two Higgs bosons are significantly larger than their mass difference it can happen that interference terms are small. An example for such a situation is the above-mentioned case of the MSSM with real soft-breaking parameters for $M_{A} \gg M_{Z}$. While for large $\tan \beta$ the widths of the two Higgs bosons can be very large, the different 
quantum numbers of the $\mathcal{C P}$-even and the $\mathcal{C P}$-odd state nevertheless forbid an interference contribution.

In determining the cross sections $\sigma(X)$ for a given signal topology $X$, HiggsBounds does the following. Suppose there are $n_{\text {Higgs }}$ neutral Higgs bosons $h_{i}$ in a given model and let the numbering be according to a mass order, i.e.

$$
m_{h_{1}} \leq m_{h_{2}} \leq \cdots \leq m_{h_{n_{\mathrm{Higgs}}}}
$$

Then, HiggsBounds works through a loop from $i=1$ to $n_{\text {Higgs }}$ and adds up all 2 production cross sections of Higgs bosons $h_{j}(j \geq i)$ which satisfy

$$
m_{h_{j}}-m_{h_{i}} \leq \Delta m_{h}(X)
$$

In the implementation, the $X$-dependence of $\Delta m_{h}$ is limited to two different values: one for LEP and one for Tevatron search topologies, represented by the variables delta_Mh_LEP and delta_Mh_TEV, respectively. Adding of Higgs signal cross sections is mainly relevant for Tevatron Higgs search analyses, where the invariant mass distributions of the potential Higgs boson decay products usually use bin sizes of the order of $10 \mathrm{GeV}$. For the LEP analyses, the Higgs mass resolution is typically around $2-3 \mathrm{GeV}[11$. For the LEP results the addition of Higgs signal cross sections is implemented in HiggsBounds only for the Higgsstrahlung process where the Higgs boson decays directly into SM particles. As default values for delta_Mh_LEP and delta_Mh_TEV we have chosen (in units of $\mathrm{GeV}$ ):

$$
\begin{aligned}
& \text { delta_Mh_TEV }=10, \\
& \text { delta_Mh_LEP }=2 .
\end{aligned}
$$

The default setting of delta_Mh_TEV for the Tevatron search channels has also been tested against known MSSM Higgs search results [23,24,25] which we could reproduce successfully with HiggsBounds within the limitations of the narrow-width approach.

In the calculation of normalised quantities, the reference cross section and branching ratio are evaluated at the average mass of the Higgs bosons contributing to a signal topology.

\section{Provided SM normalisation}

In the evaluation of the model predictions $Q_{\text {model }}(X)$ and $Q_{\text {model }}\left(\left\{X_{1}, \ldots, X_{M}\right\}\right)$ for single and multiple search topologies, respectively, to be compared with the expected and observed limits $Q_{\text {obs }}$ and $Q_{\text {expec}}$, HiggsBounds uses internally SM predictions for Higgs production cross sections and decay branching

$\overline{2}$ If the cross section for a SM combination of signal topologies is calculated, production cross sections of those Higgs bosons are only included in the sum if they also fulfil the SM-likeness criterion. 
ratios. Out of the set of implemented Higgs search results, the need for using such predictions internally arises only for Tevatron analyses. The provided SM predictions for Higgs boson production cross sections and decay branching ratios use results of HDECAY 3.303 [26] and the TEV4LHC Higgs Working Group [27] (see Table 12 below for references to the original publications). In addition, for the $b H$ production cross section we need several predictions with different kinematic cuts on the $b$ jet which are not available from [27]. We used HJET 1.1 [28] to calculate those, but checked that the uncut cross section closely resembles the result reported in [27] (see Section 3.3 for details). If the model input is given in terms of ratios of partonic cross sections, the calculation of hadronic Higgs production cross sections is facilitated by using internally SM cross section ratios which we have calculated (see Section 2.3.2 for details). If the model input is given in terms of effective couplings, we make use of results from VBFNLO [29] in order to facilitate the calculation of the Higgs production cross section via vector boson fusion (see Section 2.3.3 for details).

The rationale behind the choice of SM normalisation is that virtually all Tevatron analyses implemented in this program use these predictions when normalising their cross section limits to SM quantities. Thus, describing deviations from the SM of a new model by using the SM normalisation the experimental analyses have chosen allows for the most accurate interpretation of the limits. By using SM predictions for Higgs production cross sections which deviate from the internally used ones by a certain percentage (be it because of a different loop order, different numerical values of input parameters, renormalisation scheme or choice of parton distribution functions), a deviation of the same relative size will be caused in the quantities $Q_{\text {model }}$. The user should bear this in mind when interpreting the output of HiggsBounds.

\subsection{LEP limits}

The main Higgs boson production processes at LEP have been Higgsstrahlung and double Higgs production:

$$
e^{+} e^{-} \rightarrow h_{k} Z, \quad e^{+} e^{-} \rightarrow h_{k} h_{i} .
$$

Both processes are assumed to be mediated via $s$-channel $Z$ boson exchange in leading order of perturbation theory.

Currently, HiggsBounds uses the exclusion limits on topological cross sections obtained from the combination of all LEP2 and some LEP1 Higgs boson search results from Ref. [12] which considers the Higgs decay final states $b \bar{b}$ and $\tau^{+} \tau^{-}$. Apart from SM search topologies, this analysis also considers double Higgs production topologies, and almost all possibilities for the decay of an 


\begin{tabular}{l|l} 
search topology $X$ & reference \\
\hline$e^{+} e^{-} \rightarrow\left(h_{k}\right) Z \rightarrow(b \bar{b}) Z$ & {$[1230]$ table $14 \mathrm{~b}$} \\
$e^{+} e^{-} \rightarrow\left(h_{k}\right) Z \rightarrow\left(\tau^{+} \tau^{-}\right) Z$ & {$[1230]$ table $14 \mathrm{c}$} \\
$e^{+} e^{-} \rightarrow\left(h_{k} \rightarrow h_{i} h_{i}\right) Z \rightarrow(b \bar{b} b \bar{b}) Z$ & {$[1231]$ table 15} \\
$e^{+} e^{-} \rightarrow\left(h_{k} \rightarrow h_{i} h_{i}\right) Z \rightarrow\left(\tau^{+} \tau^{-} \tau^{+} \tau^{-}\right) Z$ & {$[1231]$ table 16} \\
$e^{+} e^{-} \rightarrow\left(h_{k} h_{i}\right) \rightarrow(b \bar{b} b \bar{b})$ & {$[1231]$ table 18} \\
$e^{+} e^{-} \rightarrow\left(h_{k} h_{i}\right) \rightarrow\left(\tau^{+} \tau^{-} \tau^{+} \tau^{-}\right)$ & {$[1231]$ table 19} \\
$e^{+} e^{-} \rightarrow\left(h_{k} \rightarrow h_{i} h_{i}\right) h_{i} \rightarrow(b \bar{b} b \bar{b}) b \bar{b}$ & {$[1231]$ table 20} \\
$e^{+} e^{-} \rightarrow\left(h_{k} \rightarrow h_{i} h_{i}\right) h_{i} \rightarrow\left(\tau^{+} \tau^{-} \tau^{+} \tau^{-}\right) \tau^{+} \tau^{-}$ & {$[1231]$ table 21} \\
$e^{+} e^{-} \rightarrow\left(h_{k} \rightarrow h_{i} h_{i}\right) Z \rightarrow(b \bar{b})\left(\tau^{+} \tau^{-}\right) Z$ & {$[31]$} \\
$e^{+} e^{-} \rightarrow\left(h_{k} \rightarrow b \bar{b}\right)\left(h_{i} \rightarrow \tau^{+} \tau^{-}\right)$ & $31]$ \\
$e^{+} e^{-} \rightarrow\left(h_{k} \rightarrow \tau^{+} \tau^{-}\right)\left(h_{i} \rightarrow b \bar{b}\right)$ & {$[31]$}
\end{tabular}

Table 1

LEP search topologies used by HiggsBounds. For each Higgs search topology which has been studied in [12], we indicate the corresponding table number. It is assumed in all tables that $m_{h_{k}}>m_{h_{i}}$. In the program, we use more fine-grained results [30 31] from the analysis [12] than have been displayed as tables in [12]. We also use equally fine-grained results for the expected limits [30 31], which have only been displayed in graphs in [12], and for the observed and expected limits of a few channels, which were not published [31].

on-shell Higgs boson into two lighter ones 3 and their subsequent decay into matter particles. Because of the limitation in [12] to $b \bar{b}$ and $\tau^{+} \tau^{-}$final states, Higgs bosons which decay predominantly invisibly cannot be well constrained by HiggsBounds at present. We will include the appropriate LEP results in future updates of the code. The search topologies included in HiggsBounds up to now are shown in Table 1 .

The limits on topological cross sections in [12] have been given in the normalisation where all Higgs decay branching ratios are equal to 1. For given Higgs mass(es), limits on the production cross sections of the individual search topologies $X$ have been derived for this normalisation in the form of scaling

$\overline{3}$ The only possibilities not analysed in [12] are the ones where more than two distinct Higgs bosons appear. For example, this occurs if a primarily produced Higgs boson $h_{k}$ decays into two different Higgs bosons $h_{j}$ and $h_{i}$, or if two Higgs bosons $h_{k} h_{j}$ are primarily produced and one of these, say $h_{k}$, decays into two Higgs bosons $h_{i}(i, j, k$ all different). We are not aware of any studies by the LEP experiments of those more general topologies. 
factors $S_{95}(X)$, defined as

$$
S_{95}(X)=\sigma_{\max }(X) / \sigma_{\text {ref }}(X)
$$

where $\sigma_{\max }$ is the largest cross section compatible with the data at $95 \%$ C.L. and $\sigma_{\text {ref }}$ is a reference cross section for the Higgs production process. The quantities $S_{95}(X)$ of [12] correspond to the quantities $Q(X)$ mentioned in the description of the program flow in Section 2.1.

For Higgsstrahlung processes, $e^{+} e^{-} \rightarrow h_{k} Z \rightarrow$ (final state) $Z$, the reference cross section $\sigma_{\text {ref }}$ is the $\mathrm{SM}$ cross section

$$
\sigma_{\text {ref }}(\text { Higgsstrahlung })=\sigma\left(e^{+} e^{-} \rightarrow H Z\right)_{\mathrm{SM}}
$$

with the SM Higgs mass $m_{H}$ chosen as $m_{h_{k}}$.

For double Higgs production processes, $e^{+} e^{-} \rightarrow H^{\prime} H \rightarrow$ (final state), there is no direct SM reference process. However, as in [12], we choose a reference cross section for a fictitious production process of two scalar particles $\left(H^{\prime}\right.$, $H)$ with masses $m_{H^{\prime}}=m_{h_{k}}$ and $m_{H}=m_{h_{i}}$ via a virtual $Z$ exchange with a standardised coupling constant

$$
g_{H^{\prime} H Z}^{\mathrm{ref}}=\frac{e}{2 s_{\mathrm{w}} c_{\mathrm{w}}}
$$

where $e$ denotes the electromagnetic coupling constant, and $s_{\mathrm{w}}$ and $c_{\mathrm{w}}$ the sine and cosine of the electroweak mixing angle, respectively. The cross section of this process in leading order is then completely determined by the Higgs masses and SM input and related to the SM Higgsstrahlung cross section via a simple phase space factor:

$$
\begin{aligned}
\sigma_{\text {ref }}\left(H^{\prime} H \text { production }\right) & =\bar{\lambda}\left(m_{H^{\prime}}, m_{H}, s\right) \sigma_{H Z}^{\mathrm{SM}}\left(m_{H}\right), \\
\bar{\lambda}\left(m_{H^{\prime}}, m_{H}, s\right) & =\frac{\lambda_{H^{\prime} H}^{3 / 2}(s)}{\lambda_{H Z}^{1 / 2}(s)\left(\lambda_{H Z}(s)+12 \frac{m_{Z}^{2}}{s}\right)}, \\
\lambda_{a b}(s) & =\left[1-\frac{\left(m_{a}+m_{b}\right)^{2}}{s}\right]\left[1-\frac{\left(m_{a}-m_{b}\right)^{2}}{s}\right] .
\end{aligned}
$$

This reference cross section coincides with the MSSM or THDM tree-level cross section for the process $e^{+} e^{-} \rightarrow h^{0} A^{0}$ if the Higgs mixing-angle dependent factor $\cos (\beta-\alpha)$ is divided out and $m_{A^{0}}$ and $m_{h^{0}}$ are chosen as $m_{H^{\prime}}$ and $m_{H}$.

In order to apply the LEP Higgs constraints implemented in HiggsBounds to a model, the user has to provide model information which allows model predictions to be calculated for all Higgs search topologies $X$ listed in Table 
1.

$$
Q_{\text {model }}(X)=\frac{\sigma_{\text {model }}(P(X)) \mathrm{BR}_{\text {model }}(F(X))}{\sigma_{\text {ref }}(P(X))},
$$

where the symbol $P(X)$ denotes the corresponding Higgs production process and $F(X)$ the corresponding Higgs decay chain, e.g. $h_{k} \rightarrow b \bar{b}$ or $h_{k} \rightarrow h_{i} h_{i} \rightarrow$ $b \bar{b} \tau^{+} \tau^{-}$. The quantity $Q_{\text {model }}(X)$ is compared with the experimental limits $Q_{\text {expec }}(X)$ and $Q_{\text {obs }}(X)$ according to the description in Section 2.1. Currently, HiggsBounds allows model information to be provided in three ways.

\subsubsection{LEP input (HiggsBounds Input Option part and hadr): cross section and branching ratios}

In order to be able to apply the implemented LEP limits to the model under study, the user is asked to provide model predictions for the Higgs boson masses $m_{h_{k}}$ (in units of $\mathrm{GeV}$ ), for the branching ratios

$$
\mathrm{BR}_{\text {model }}\left(h_{i} \rightarrow b \bar{b}\right), \quad \mathrm{BR}_{\text {model }}\left(h_{i} \rightarrow \tau^{+} \tau^{-}\right), \quad \mathrm{BR}_{\text {model }}\left(h_{k} \rightarrow h_{i} h_{i}\right),
$$

and for the ratios of cross sections

$$
\frac{\sigma_{\text {model }}\left(e^{+} e^{-} \rightarrow h_{k} Z\right)}{\sigma_{\text {ref }}\left(e^{+} e^{-} \rightarrow H Z\right)}, \quad \frac{\sigma_{\text {model }}\left(e^{+} e^{-} \rightarrow h_{k} h_{i}\right)}{\sigma_{\text {ref }}\left(e^{+} e^{-} \rightarrow H^{\prime} H\right)},
$$

for $k, i \in\left\{1, \ldots, n_{\mathrm{Higgs}}\right\}$.

With this information, HiggsBounds obtains model predictions $Q_{\text {model }}(X)$ for all topologies $X$ specified in Table 1. For example, the $Q_{\text {model }}$ value for the channel $e^{+} e^{-} \rightarrow h_{1} Z \rightarrow(b \bar{b}) Z$ is given by (suppressing the initial state in the notation):

$$
Q_{\text {model }}\left(\left(h_{1}\right) Z \rightarrow(b \bar{b}) Z\right)=\frac{\sigma_{\text {model }}\left(h_{1} Z\right)}{\sigma_{\text {ref }}(H Z)} \mathrm{BR}_{\text {model }}\left(h_{1} \rightarrow b \bar{b}\right),
$$

with $m_{H}=m_{h_{1}}$, and similarly for the channel $e^{+} e^{-} \rightarrow\left(h_{2} \rightarrow h_{1} h_{1}\right) Z \rightarrow$ $(b \bar{b} b \bar{b}) Z$ it is:

$$
\begin{aligned}
& Q_{\text {model }}\left(\left(h_{2} \rightarrow h_{1} h_{1}\right) Z \rightarrow(b \bar{b} b \bar{b}) Z\right)= \\
& \quad \frac{\sigma_{\text {model }}\left(h_{2} Z\right)}{\sigma_{\text {ref }}(H Z)} \mathrm{BR}_{\text {model }}\left(h_{2} \rightarrow h_{1} h_{1}\right)\left(\mathrm{BR}_{\text {model }}\left(h_{1} \rightarrow b \bar{b}\right)\right)^{2},
\end{aligned}
$$

with $m_{H}=m_{h_{2}}$.

The HiggsBounds Input Option hadr amounts to exactly the same LEP related input as described above. It differs for the Tevatron related input (see Section 2.3). 
If the model under study predicts kinematical distributions which differ significantly from the distributions of the reference processes (18) or (20), the exclusion result, returned by HiggsBounds, can only be considered an estimate 4

\subsubsection{LEP input (HiggsBounds Input Option effC): effective couplings}

The user is asked to provide model predictions for:

$$
\begin{aligned}
& m_{h_{k}}, \Gamma_{\text {tot }}\left(h_{k}\right), \quad\left(\frac{g_{h_{k} Z Z}^{\text {model }}}{g_{H Z Z}^{\mathrm{SM}}}\right)^{2}, \quad\left(\frac{g_{h_{k} h_{i} Z}^{\text {model }}}{g_{H^{\prime} H Z}^{\text {ref }}}\right)^{2}, \quad\left(\frac{g_{h_{k} f \bar{f}, \text { eff }}^{\text {mode }}}{g_{H f \bar{f}}^{\mathrm{SM}}}\right)^{2}, \\
& \operatorname{BR}_{\text {model }}\left(h_{k} \rightarrow h_{i} h_{i}\right),
\end{aligned}
$$

for $k, i \in\left\{1, \ldots, n_{\text {Higgs }}\right\}$ and $f \in\{b, \tau\}$. The normalised effective squared coupling of the Higgs boson fermion interaction is defined as an approximation for the ratio of partial decay widths,

$$
\left(\frac{g_{h_{k} f \bar{f}, \mathrm{eff}}^{\text {model }}}{g_{H f \bar{f}}^{\mathrm{SM}}}\right)^{2}:=\left.\frac{\Gamma_{h_{k} \rightarrow f \bar{f}}^{\text {model }}\left(m_{h_{k}}\right)}{\Gamma_{H \rightarrow f \bar{f}}^{\mathrm{SM}}\left(m_{H}\right)}\right|_{m_{H}=m_{h_{k}}},
$$

and the reference coupling constants are defined by Eq. (19) and by

$$
\begin{aligned}
\left(g_{H Z Z}^{\mathrm{SM}}\right)^{2} & =\left(\frac{e}{s_{\mathrm{w}}} \frac{m_{W}}{c_{\mathrm{w}}^{2}}\right)^{2}, \\
\left(g_{H f \bar{f}}^{\mathrm{SM}}\right)^{2} & =\left(\frac{1}{2} \frac{e}{s_{\mathrm{w}}} \frac{m_{f}}{m_{W}}\right)^{2},
\end{aligned}
$$

where $m_{W}$ and $m_{f}$ denote the masses of the $W$ boson and fermion $f$, respectively.

For the purpose of running HiggsBounds, only the ratio of partial widths corresponding to the right-hand-side of Eq. (25) is needed. In order to completely specify effective couplings for neutral Higgs boson interactions with fermions, in general a scalar and a pseudoscalar part of the coupling is needed. The Feynman rule for the coupling of a generic neutral Higgs boson $h$ to fermions can be written as:

$$
G(h f \bar{f})=i\left(g_{s} \mathbf{1}+i g_{p} \gamma_{5}\right)
$$

4 In such a case, the general cross section limits given by the experimental collaborations would have to be replaced by a dedicated experimental analysis of a particular model giving rise to these kinematical distributions, taking into account detailed information about the detector response to the signal events. 
where $g_{s}$ and $g_{p}$ are real-valued coupling constants, and $\mathbf{1}$ and $\gamma_{5}$ are the usual matrices in Dirac space. A scalar particle, like the SM Higgs boson, has $g_{p}=0$ and a pseudoscalar particle has $g_{s}=0$. If the user has the couplings $g_{s}$ and $g_{p}$ available, the user may calculate the normalised effective squared couplings, specified in Eq. (25), by using the relation:

$$
\left(\frac{g_{h_{k} f \bar{f}, \text { eff }}^{\text {model }}}{g_{H f \bar{f}}^{\mathrm{SM}}}\right)^{2}=\left(\frac{g_{s, h_{k} f \bar{f}}^{\text {model }}}{g_{H f \bar{f}}^{\mathrm{SM}}}\right)^{2}+\left(\frac{g_{p, h_{k} f \bar{f}}^{\text {model }}}{g_{H f \bar{f}}^{\mathrm{SM}}}\right)^{2} \frac{1}{\beta_{f}^{2}\left(m_{h_{k}}\right)},
$$

with

$$
\beta_{f}\left(m_{h_{k}}\right)=\sqrt{1-\frac{4 m_{f}^{2}}{m_{h_{k}}^{2}}}
$$

The extra factor of $\beta_{f}^{-2}$ in Eq. (29) appears, because the partial width for a scalar particle decaying into $f \bar{f}$ is proportional to $\beta_{f}^{3}$, while for a pseudoscalar particle it is proportional to $\beta_{f}$.

The user may also avoid the explicit use of the reference coupling, defined in Eq. (26), by using the relation

$$
\left(\frac{g_{h_{k} Z Z}^{\text {model }}}{g_{H Z Z}^{\mathrm{SM}}}\right)^{2}=\left.\frac{\Gamma_{h_{k} \rightarrow Z^{(\star)} Z^{(\star)}}^{\text {model }}\left(m_{h_{k}}\right)}{\Gamma_{H \rightarrow Z^{(\star)} Z^{(\star)}}^{\mathrm{SM}}\left(m_{H}\right)}\right|_{m_{H}=m_{h_{k}}},
$$

where the asterisk in brackets indicates the possible off-shellness of the decay products. Knowledge of the off-shell decay matrix elements allows to use relation (30) for masses $m_{h_{k}}$ smaller than $2 M_{Z}$.

From the information specified in Eq. (24), HiggsBounds calculates the LEP part of the cross section and branching ratios required in Input Option part in the effective coupling approximation:

$$
\begin{aligned}
\frac{\sigma_{\text {model }}\left(e^{+} e^{-} \rightarrow h_{k} Z\right)}{\sigma_{\text {ref }}\left(e^{+} e^{-} \rightarrow H Z\right)} & =\left(\frac{g_{h_{k} Z Z}^{\text {model }}}{g_{H Z Z}^{\mathrm{SM}}}\right)^{2}, \\
\frac{\sigma_{\text {model }}\left(e^{+} e^{-} \rightarrow h_{k} h_{i}\right)}{\sigma_{\text {ref }}\left(e^{+} e^{-} \rightarrow h_{k} h_{i}\right)} & =\left(\frac{g_{H^{\prime} H Z}^{\text {model }}}{g_{H^{\prime} H Z}^{\text {ref }}}\right)^{2}, \\
\operatorname{BR}_{\text {model }}\left(h_{k} \rightarrow f \bar{f}\right) & =\left.\operatorname{BR}_{\mathrm{SM}}(H \rightarrow f \bar{f})\left(m_{H}\right) \frac{\Gamma_{\text {tot }}^{\mathrm{SM}}\left(m_{H}\right)}{\Gamma_{\text {tot }}\left(h_{k}\right)}\right|_{m_{H}=m_{h_{k}}} \times \\
& \times\left(\frac{g_{h_{k} f \bar{f}, \text { eff }}^{\text {model }}}{g_{H f \bar{f}}^{\mathrm{SM}}}\right)^{2},
\end{aligned}
$$

for $f \in\{b, \tau\}$. 
From this point, the evaluation of the quantities $Q_{\text {model }}(X)$ can proceed exactly as exemplified in Section 2.2.1.

As far as the implemented LEP constraints are concerned, effective couplings just rescale all kinematical distributions of the reference processes (18) or (20) by common factors. Thus, for the class of models where the Higgs sector can be faithfully parametrised in this way, the kinematical distributions correspond to those of the reference processes. Consequently, limits on topological cross sections can exactly be applied.

\subsection{Tevatron limits}

The main SM Higgs boson production processes at the proton-anti-proton collider Tevatron at Fermilab are:

- Gluon fusion: $p \bar{p} \rightarrow H+\cdots$ via $g g \rightarrow H$,

- Higgsstrahlung: $p \bar{p} \rightarrow H V+\cdots$ via $q \bar{q}^{\prime} \rightarrow V H\left(V=Z, W^{ \pm}\right)$,

- Vector boson fusion (VBF) $p \bar{p} \rightarrow H j j+\cdots$ via $q q^{\prime} \rightarrow V^{\star} V^{\star \star} q^{\prime \prime} q^{\prime \prime \prime} \rightarrow H q^{\prime \prime} q^{\prime \prime \prime}$,

- Higgs production associated with heavy quarks: $p \bar{p} \rightarrow H t \bar{t}(H b \bar{b})+\cdots$,

where the ellipses indicate the hadronic remainder of the reaction which we will suppress in our notation below.

Currently, the Tevatron experiments CDF and D $\varnothing$ provide separate limits on cross sections of individual Higgs search topologies $X$ and limits on combinations of search topologies according to SM proportions. In particular, combinations of Higgs search results from both experiments are, so far, only available for a SM-like Higgs boson. The results implemented in HiggsBounds correspond to the Higgs search topologies that have been analysed by CDF and DØ, using Tevatron Run II data, listed in Tables 2 and 3 . New results provided by $\mathrm{CDF}$ and $\mathrm{D} \varnothing$ will be implemented once they appear, thus keeping HiggsBounds up-to-date.

In the absence of a Higgs signal, the Tevatron experiments obtain cross section limits from their measurements: $\sigma_{\max }(X)$ is the highest cross section for a given assumed Higgs mass $m_{H}$ which is compatible with the only-background hypothesis at 95\% C.L. Therefore, if a particular model of the Higgs sector predicts a cross section $\sigma_{\text {model }}(X)$ for a Higgs boson with mass $m_{H}$, which is higher than the limit, i.e.

$$
\sigma_{\text {model }}(X)>\sigma_{\max }(X)
$$

then the probability that such a prediction is consistent with observation is less than $5 \%$ and the model parameter point will be called excluded with (at 


\begin{tabular}{l|l} 
search topology $X$ (analysis) & reference \\
\hline$p \bar{p} \rightarrow Z H \rightarrow l^{+} l^{-} b \bar{b}\left(\mathrm{CDF}\right.$ with $\left.1.0 \mathrm{fb}^{-1}\right)$ & {$[32]^{\star}$} \\
$p \bar{p} \rightarrow Z H \rightarrow l^{+} l^{-} b \bar{b}\left(\mathrm{CDF}\right.$ with $\left.2.4 \mathrm{fb}^{-1}\right)$ & {$[33]$} \\
$p \bar{p} \rightarrow Z H \rightarrow l^{+} l^{-} b \bar{b}\left(\mathrm{D} \varnothing\right.$ with $\left.2.3 \mathrm{fb}^{-1}\right)$ & {$[34]$} \\
$p \bar{p} \rightarrow W H \rightarrow l \nu b \bar{b}\left(\mathrm{D} \varnothing\right.$ with $\left.1.7 \mathrm{fb}^{-1}\right)$ & {$[35]$} \\
$p \bar{p} \rightarrow W H \rightarrow l \nu b \bar{b}\left(\mathrm{CDF}\right.$ with $\left.2.7 \mathrm{fb}^{-1}\right)$ & {$[36]$} \\
$p \bar{p} \rightarrow W H \rightarrow W^{+} W^{-} W^{ \pm}\left(\mathrm{D} \varnothing\right.$ with $\left.1.0 \mathrm{fb}^{-1}\right)$ & {$[37]$} \\
$p \bar{p} \rightarrow W H \rightarrow W^{+} W^{-} W^{ \pm}\left(\mathrm{CDF}\right.$ with $\left.1.9 \mathrm{fb}^{-1}\right)$ & {$[38]$} \\
$p \bar{p} \rightarrow H \rightarrow W^{+} W^{-} \rightarrow l^{+} l^{-}\left(\mathrm{D} \varnothing\right.$ with $\left.3.0 \mathrm{fb}^{-1}\right)$ & {$[39]$} \\
$p \bar{p} \rightarrow H \rightarrow W^{+} W^{-} \rightarrow l^{+} l^{\prime-}\left(\mathrm{CDF}^{\mathrm{with}} 3.0 \mathrm{fb}^{-1}\right)$ & {$[40]^{\star}$} \\
$p \bar{p} \rightarrow H \rightarrow \gamma \gamma\left(\mathrm{D} \varnothing\right.$ with $\left.1.1 \mathrm{fb}^{-1}\right)$ & {$[41]^{\star}$} \\
$p \bar{p} \rightarrow H \rightarrow \gamma \gamma\left(\mathrm{D} \varnothing\right.$ with $\left.2.68 \mathrm{fb}^{-1}\right)$ & {$[42]$} \\
$p \bar{p} \rightarrow H \rightarrow \tau^{+} \tau^{-}\left(\mathrm{D} \varnothing\right.$ with $\left.1.0 \mathrm{fb}^{-1}\right)$ & {$[24]^{\star}$} \\
$p \bar{p} \rightarrow H \rightarrow \tau^{+} \tau^{-}\left(\mathrm{CDF}\right.$ with $\left.1.8 \mathrm{fb}^{-1}\right)$ & {$[25]$} \\
$p \bar{p} \rightarrow b H, H \rightarrow b \bar{b}\left(\mathrm{CDF}\right.$ with $\left.1.9 \mathrm{fb}^{-1}\right)$ & {$[22]$} \\
$p \bar{p} \rightarrow b H, H \rightarrow b \bar{b}\left(\mathrm{D} \varnothing\right.$ with $\left.1.0 \mathrm{fb}^{-1}\right)$ & {$[23]^{\star}$} \\
$p \bar{p} \rightarrow b H, H \rightarrow b \bar{b}\left(\mathrm{D} \varnothing\right.$ with $\left.2.6 \mathrm{fb}^{-1}\right)$ & {$[43]$} \\
$\mathrm{T} \rightarrow H$
\end{tabular}

Table 2

Tevatron analyses for single search topologies, the results of which are used by HiggsBounds. The leptons $l$ and $l^{\prime}$ can be an electron or a muon. References marked by an asterisk $(\star)$ refer to analyses which have been published or submitted for publication.

least) $95 \%$ C.L. While some Tevatron Higgs search results are presented in the form of absolute cross section limits, $\sigma_{\max }(X)$, most of the results are presented as limits on signal cross sections normalised to the corresponding $\mathrm{SM}$ cross section, $\sigma_{\mathrm{SM}}(X) 5$ In the latter case, the exclusion condition (34) becomes:

$$
\frac{\sigma_{\text {model }}(X)}{\sigma_{\mathrm{SM}}(X)}>\frac{\sigma_{\max }(X)}{\sigma_{\mathrm{SM}}(X)} .
$$

In order to test the exclusion of a given model by CDF or D $\varnothing$ results according to the procedure outlined in Section 2.1, the user needs to provide model

$\overline{5}$ So far, no Tevatron Higgs search results have appeared which would require to use a normalisation to non-SM cross sections. 


\begin{tabular}{|c|c|}
\hline search topologies $\{X\}$ (analysis) & reference \\
\hline$p \bar{p} \rightarrow W H / Z H \rightarrow b \bar{b}+E_{T}^{\text {miss. }}\left(\right.$ CDF with $\left.2.3 \mathrm{fb}^{-1}\right)$ & 44 \\
\hline$p \bar{p} \rightarrow W H / Z H \rightarrow b \bar{b}+E_{T}^{\text {miss. }}\left(\mathrm{D} \varnothing\right.$ with $\left.2.1 \mathrm{fb}^{-1}\right)$ & 45 \\
\hline$p \bar{p} \rightarrow H / H W / H Z / H$ via $\mathrm{VBF}, H \rightarrow \tau^{+} \tau^{-}\left(\mathrm{CDF}\right.$ with $\left.2.0 \mathrm{fb}^{-1}\right)$ & 46 \\
\hline Combined SM analysis (CDF \& DØ with $0.9-1.9 \mathrm{fb}^{-1}$ ) & [47] \\
\hline Combined SM analysis (CDF \& DØ with $1.0-2.4 \mathrm{fb}^{-1}$ ) & 48 \\
\hline Combined SM analysis (CDF \& DØ with $3.0 \mathrm{fb}^{-1}$ ) & 49 \\
\hline
\end{tabular}

Table 3

$C D F$ and $D \varnothing$ analyses which are contained in HiggsBounds and which combine search topologies according to SM proportions.

predictions which allow, for each relevant Higgs boson search topology $X$, either absolute cross sections,

$$
Q_{\text {model }}(X)=\sigma_{\text {model }}(P(X)) \mathrm{BR}_{\text {model }}(F(X))
$$

or cross section ratios,

$$
Q_{\text {model }}(X)=\frac{\sigma_{\text {model }}(P(X)) \mathrm{BR}_{\text {model }}(F(X))}{\sigma_{\mathrm{SM}}(P(X)) \mathrm{BR}_{\mathrm{SM}}(F(X))}
$$

of hadronic processes to be calculated, where the symbol $P(X)$ denotes the corresponding Higgs production process and $F(X)$ the corresponding Higgs decay chain. The program allows both variants to be calculated from the same input, as the SM predictions for Higgs production processes and decay branching ratios are internally available. In Eqs. (36) and (37), we have already used the implicit assumption that Higgs bosons have narrow width, for which the CDF and D $\varnothing$ limits we employ are valid. We should also stress again that the limits are based on analyses which assume that the backgrounds to the signal process under study are similar to the backgrounds predicted by the SM. For that reason, the branching ratios for the decays of the $W^{ \pm}$or the $Z$ for a particular individual Higgs search topology $X$ are assumed to cancel in the ratio (37).

For the calculation of signal cross section predictions where several Higgs search topologies $\left\{X_{1}, \ldots, X_{M}\right\}$ are combined (all cases listed in Table [3), the definition in Eq. (5) applies. In the narrow-width approximation, this definition reads:

$$
Q_{\text {model }}\left(\left\{X_{1}, \ldots X_{M}\right\}\right)=\frac{\sum_{n=1}^{M} \sigma_{\text {model }}\left(P\left(X_{n}\right)\right) \mathrm{BR}_{\text {model }}\left(F\left(X_{n}\right)\right)}{\sum_{n=1}^{M} \sigma_{\mathrm{SM}}\left(P\left(X_{n}\right) \mathrm{BR}_{\mathrm{SM}}\left(F\left(X_{n}\right)\right)\right.}
$$


The quantities $Q_{\text {model }}$ are to be compared with the experimental limits $Q_{\text {expec }}$ and $Q_{\text {obs }}$ according to the description in Section 2.1. There are three options, which allow the user to provide HiggsBounds with the necessary model information in order to calculate the quantities $Q_{\text {model }}$. These are described in the following.

\subsubsection{Tevatron input (HiggsBounds Input Option hadr): hadronic cross sec- tion and branching ratios}

In order to make use of all the implemented Tevatron limits, the user is asked

to provide model predictions for the Higgs boson masses $m_{h_{k}}$ (in units of GeV), for the branching ratios

$$
\begin{array}{ll}
\operatorname{BR}_{\text {model }}\left(h_{k} \rightarrow b \bar{b}\right), & \operatorname{BR}_{\text {model }}\left(h_{k} \rightarrow \tau^{+} \tau^{-}\right), \\
\operatorname{BR}_{\text {model }}\left(h_{k} \rightarrow W^{+} W^{-}\right), & \operatorname{BR}_{\text {model }}\left(h_{k} \rightarrow \gamma \gamma\right),
\end{array}
$$

and for the ratios of the hadronic cross sections

$$
\begin{array}{lll}
\frac{\sigma_{\text {model }}\left(p \bar{p} \rightarrow h_{k} Z\right)}{\sigma_{\mathrm{SM}}(p \bar{p} \rightarrow H Z)}, & \frac{\sigma_{\text {model }}\left(p \bar{p} \rightarrow h_{k} W^{ \pm}\right)}{\sigma_{\mathrm{SM}}\left(p \bar{p} \rightarrow H W^{ \pm}\right)}, & \frac{\sigma_{\text {model }}\left(p \bar{p} \rightarrow h_{k} \text { via } \mathrm{VBF}\right)}{\sigma_{\mathrm{SM}}(p \bar{p} \rightarrow H \text { via } \mathrm{VBF})} \\
\frac{\sigma_{\text {model }}\left(p \bar{p} \rightarrow h_{k}\right)}{\sigma_{\mathrm{SM}}(p \bar{p} \rightarrow H)}, & \frac{\sigma_{\text {model }}\left(p \bar{p} \rightarrow h_{k} b\right)}{\sigma_{\mathrm{SM}}(p \bar{p} \rightarrow H b)}
\end{array}
$$

for $k \in\left\{1, \ldots, n_{\text {Higgs }}\right\}$.

With this information and the internally available SM predictions for Higgs production cross sections and decay branching ratios, HiggsBounds calculates all values $Q_{\text {model }}$, according to Eqs. (36), (37) or (38), which are needed in order to test the chosen model against the implemented Tevatron results. Note that the branching ratios $\mathrm{BR}_{\text {model }}\left(h_{k} \rightarrow h_{i} h_{i}\right)$ are currently not used in comparisons with Tevatron results, because, so far, no Tevatron analyses have appeared which consider such decays.

While hadr is the most widely applicable input option, it can also be the one that is most difficult for the user to provide, as this option requires the user to provide predictions for hadronic cross sections, which involve a convolution with parton distribution functions. Therefore, the program offers the Input Option part, which requires only ratios of partonic cross sections. This should in general be more convenient for the user. However, this input option is applicable to a narrower class of models than Input Option hadr.

If the model under study predicts kinematical distributions which differ significantly from the distributions of the reference SM processes, the exclusion result, returned by HiggsBounds, can only be considered an estimate (as discussed at the end of Section 2.2.1). 


\subsubsection{Tevatron input (HiggsBounds Input Option part): partonic cross sec-} tion and branching ratios

The user is asked to provide model predictions for the Higgs boson masses $m_{h_{k}}$ (in units of $\mathrm{GeV}$ ), for the branching ratios

$$
\begin{array}{ll}
\operatorname{BR}_{\text {model }}\left(h_{k} \rightarrow b \bar{b}\right), & \operatorname{BR}_{\text {model }}\left(h_{k} \rightarrow \tau^{+} \tau^{-}\right), \\
\operatorname{BR}_{\text {model }}\left(h_{k} \rightarrow W^{+} W^{-}\right), & \operatorname{BR}_{\text {model }}\left(h_{k} \rightarrow \gamma \gamma\right),
\end{array}
$$

for the following ratios of partonic cross sections

$$
\begin{array}{lll}
\frac{\hat{\sigma}_{\text {model }}\left(g g \rightarrow h_{k}\right)}{\hat{\sigma}_{\mathrm{SM}}(g g \rightarrow H)}, & \frac{\hat{\sigma}_{\text {model }}\left(b \bar{b} \rightarrow h_{k}\right)}{\hat{\sigma}_{\mathrm{SM}}(b \bar{b} \rightarrow H)}, & \frac{\hat{\sigma}_{\text {model }}\left(b g \rightarrow h_{k} b\right)}{\hat{\sigma}_{\mathrm{SM}}(b g \rightarrow H b)}, \\
\frac{\hat{\sigma}_{\text {model }}\left(q \bar{q}^{\prime} \rightarrow h_{k} W^{+}\right)}{\hat{\sigma}_{\mathrm{SM}}\left(q \bar{q}^{\prime} \rightarrow H W^{+}\right)}, & \frac{\hat{\sigma}_{\text {model }}\left(q^{\prime} \bar{q} \rightarrow h_{k} W^{-}\right)}{\hat{\sigma}_{\mathrm{SM}}\left(q^{\prime} \bar{q} \rightarrow H W^{-}\right)}, & \left(q, q^{\prime}\right) \in\{(u, d),(c, s)\}, \\
\frac{\hat{\sigma}_{\text {model }}\left(q \bar{q} \rightarrow h_{k} Z\right)}{\hat{\sigma}_{\mathrm{SM}}(q \bar{q} \rightarrow H Z)}, & q \in\{u, d, c, s, b\}, &
\end{array}
$$

and for the hadronic cross section ratio

$$
\frac{\sigma_{\text {model }}\left(p \bar{p} \rightarrow h_{k} \text { via } \mathrm{VBF}\right)}{\sigma_{\mathrm{SM}}(p \bar{p} \rightarrow H \text { via } \mathrm{VBF})},
$$

for $k \in\left\{1, \ldots, n_{\mathrm{Higgs}}\right\}$.

With this information and some internally provided functions (described below) HiggsBounds can calculate the same input quantities as required for Input Option hadr (subject to some approximations, as described below). It is assumed that the model cross sections for the processes $\bar{b} g \rightarrow h_{k} \bar{b}$ and $b g \rightarrow h_{k} b$ are equal to each other.

Hadronic Higgs production cross sections can be written as a sum of cross sections of partonic processes $\hat{\sigma}_{n m \rightarrow H+y}\left(\hat{s}, m_{H}\right)$ convoluted by parton luminosity functions:

$$
\begin{aligned}
\sigma\left(p \bar{p} \rightarrow H+y, m_{H}\right) & =\int_{\tau_{0}}^{1} d \tau \sum_{\{n, m\}} \frac{d \mathcal{L}_{n m}^{p \bar{p}}}{d \tau} \hat{\sigma}_{n m \rightarrow H+y}\left(\hat{s}=\tau S, m_{H}\right), \\
& =: \sum_{\{n, m\}} \sigma\left(p \bar{p} \rightarrow n m \rightarrow H+y, m_{H}\right) .
\end{aligned}
$$

Here, the sum includes all distinct parton pairs only once. The centre-ofmass energy of the colliding proton $(p)$ and anti-proton $(\bar{p})$ is assumed to be $\sqrt{S}=1.96 \mathrm{TeV}$. The symbol $y$ indicates possible further particles in the

hard partonic process, like the $W^{ \pm}$or $Z$. The parton luminosity functions are 
defined as

$$
\frac{d \mathcal{L}_{n m}^{A B}}{d \tau}=\int_{\tau}^{1} \frac{d x}{x} \frac{1}{1+\delta_{n m}}\left[f_{n / A}\left(x, \mu_{F}\right) f_{m / B}\left(\frac{\tau}{x}, \mu_{F}\right)+f_{m / A}\left(x, \mu_{F}\right) f_{n / B}\left(\frac{\tau}{x}, \mu_{F}\right)\right],
$$

where $f_{n / A}\left(x, \mu_{F}\right)$ denotes the density of partons of type $n$ in the hadron $A$ carrying a fraction $x$ of the hadron's momentum at the scale $\mu_{F}$ and $(A, B)=$ $(p, \bar{p})$ for the Tevatron. The lower bound of the $\tau$-integration $\left(\tau_{0}\right)$ is determined by the minimal invariant mass of the parton system, $\hat{s}_{0}=\tau_{0} S$. The ratio $Q_{\text {model }}(X)$ of Eq. (37) factorises into a ratio of branching ratio predictions and a ratio of hadronic production cross section predictions. The calculation of the ratio of branching ratios is straightforward. In the following, we show an approximate relation (that in special cases is exact), which allows to calculate the ratios of hadronic cross sections for the Higgs production process $P$,

$$
R_{\sigma}(P):=\frac{\sigma_{\text {model }}(P)}{\sigma_{\mathrm{SM}}(P)}
$$

from the knowledge of ratios of partonic cross sections. Under the assumption that the ratio of the partonic cross sections,

$$
R_{n m}^{H+y}\left(\hat{s}, m_{H}\right):=\frac{\hat{\sigma}_{n m \rightarrow H+y}^{\text {model }}\left(\hat{s}, m_{H}\right)}{\hat{\sigma}_{n m \rightarrow H+y}^{\mathrm{SM}}\left(\hat{s}, m_{H}\right)},
$$

has, at most, a mild dependence on the parton-system centre-of-mass energy squared, $\hat{s}$, we can write Eq. (43) as

$$
R_{\sigma}(P) \approx \sum_{\{n, m\}} R_{n m}^{H+y}\left(\hat{s}_{0}, m_{H}\right) \frac{\sigma_{\mathrm{SM}}\left(p \bar{p} \rightarrow n m \rightarrow H+y, m_{H}\right)}{\sigma_{\mathrm{SM}}\left(p \bar{p} \rightarrow H+y, m_{H}\right)}
$$

with $\hat{s}_{0}$ denoting the partonic production threshold, $\hat{s}_{0}=\left(m_{H}+m_{y}\right)^{2}$, with $m_{y}=0$ in the case of single Higgs boson production. In this formula, only ratios of SM hadronic cross sections are needed, which we provide in our program.

In order to provide the cross section ratios in Eq. (45) with state-of-the-art theoretical accuracy, we pursue the following strategy. The K-factors (NNLO $\mathrm{QCD}+\mathrm{NLO} \mathrm{EW})$ for the $W H$ and $Z H$ production cross sections at the Tevatron are, to a very good approximation, independent of the generation of the initial state quarks and thus drop out in the ratios. The only non-trivial case is the single Higgs production which has contributions from gluon fusion and $b \bar{b}$ annihilation. For the calculation of the gluon fusion cross section, we assume a constant NNLO QCD K-factor of 3.6 [51] in the relevant range of $70 \mathrm{GeV}<m_{H}<300 \mathrm{GeV}$. In this range, our assumption is consistent with the $m_{H}$-dependent $\mathrm{K}$-factor reported in [51] within the uncertainty estimate also given in [51]. In order to obtain an approximate NNLO QCD cross section 

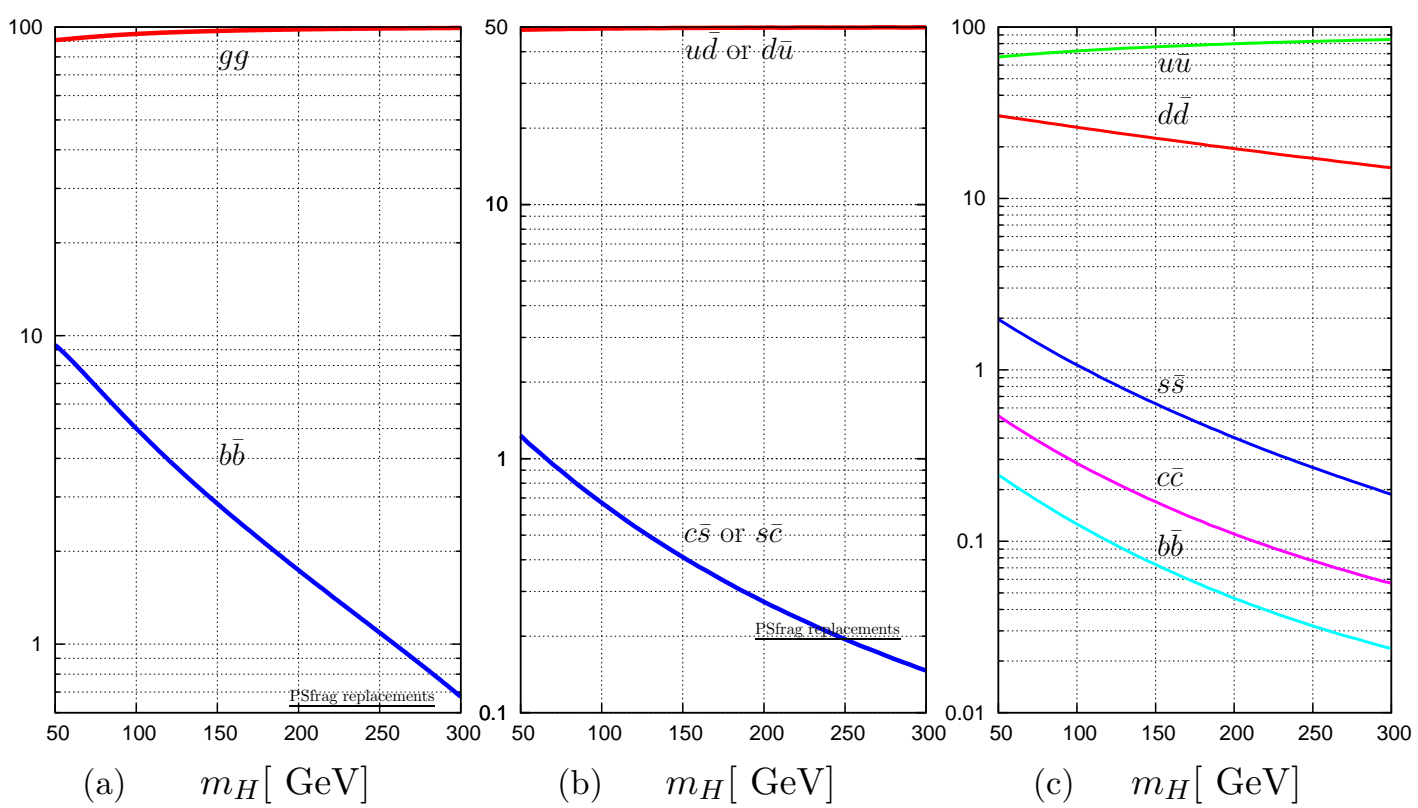

Fig. 1. Relative contributions (in \%) of different SM partonic processes to cross sections of hadronic processes: (a) single Higgs production, (b) $H W^{ \pm}$production and (c) $H Z$ production.

prediction for the $\mathrm{b}$ b-bar annihilation process, we used the fact that this cross section is known to be well approximated by the LO prediction in the MS-bar scheme with the factorisation and renormalisation scale choice of $m_{H} / 4$ [52]. The SM cross section ratios from Eq. (45) are then obtained by normalising the cross sections for one parton configuration to the appropriate sum of cross sections. As a cross-check, we confirmed that our results for Higgs production cross sections are in good agreement with the compilation of results by the TEV4LHC working group [27]. Fig. 1] shows our result: the relative contributions from different parton configurations to the total hadronic cross section for single Higgs production, $H W^{ \pm}$production and $H Z$ production in the range relevant for the implemented Tevatron analyses.

Relation (45) becomes an exact identity for all models which differ from the SM only by having different values for coupling constants, i.e. where the ratios $R_{n m}^{H+y}$ become $\hat{s}$-independent. Moreover, for models which give $\hat{s}$-dependent ratios $R_{n m}^{H+y}$, Eq. (45) can still be a good approximation because the luminosity functions $\frac{d \mathcal{L}_{n m}^{p \bar{p}}}{d \tau}$ are steeply falling functions of $\tau$ and therefore strongly favour the threshold regions of the convolution integrals. However, in this case, the user should make sure that Eq. (45) is indeed a good approximation for the model under study before using it. In case of doubt, one can always resort to Input Option hadr.

As an example, we show here how the normalised model cross section $Q_{\text {model }}(X)$ is obtained on the basis of Eq. (45) for the search topology $p \bar{p} \rightarrow H \rightarrow$ 
$W^{+} W^{-} \rightarrow l^{+} l^{--}$of Table 2 for a model Higgs boson $h_{k}$ :

$$
\begin{aligned}
Q_{\text {model }}(X)= & \left\{\left(\frac{\hat{\sigma}_{g g \rightarrow h_{k}}^{\text {model }}\left(\hat{s}_{0}, m_{h_{k}}\right)}{\hat{\sigma}_{g g \rightarrow h_{k}}^{\mathrm{SM}}\left(\hat{s}_{0}, m_{H}\right)} \frac{\sigma_{\mathrm{SM}}\left(p \bar{p} \rightarrow g g \rightarrow H, m_{H}\right)}{\sigma_{\mathrm{SM}}\left(p \bar{p} \rightarrow H, m_{H}\right)}\right.\right. \\
& \left.+\frac{\hat{\sigma}_{b \bar{b} \rightarrow h_{k}}^{\text {model }}\left(\hat{s}_{0}, m_{h_{k}}\right)}{\hat{\sigma}_{b \bar{b} \rightarrow h_{k}}^{\mathrm{SM}}\left(\hat{s}_{0}, m_{H}\right)} \frac{\sigma_{\mathrm{SM}}\left(p \bar{p} \rightarrow b \bar{b} \rightarrow H, m_{H}\right)}{\sigma_{\mathrm{SM}}\left(p \bar{p} \rightarrow H, m_{H}\right)}\right) \times \\
& \left.\times \frac{\mathrm{BR}_{h_{k} \rightarrow W^{+} W^{-}}^{\text {model }}\left(m_{h_{k}}\right)}{\mathrm{BR}_{H \rightarrow W^{+} W^{-}}^{\mathrm{SM}}\left(m_{H}\right)}\right\}\left.\right|_{m_{H}=m_{h_{k}}} .
\end{aligned}
$$

If the model under study predicts kinematical distributions which differ significantly from the distributions of the reference SM processes, the exclusion result, returned by HiggsBounds, can only be considered an estimate. Further comments regarding this limitation from the end of Section 2.2.1 apply here too.

\subsubsection{Tevatron input (HiggsBounds Input Option effC): effective couplings}

The user is asked to provide model predictions for the Higgs boson masses $m_{h_{k}}$ and total decay widths $\Gamma_{\text {tot }}\left(h_{k}\right)$ (in units of $\mathrm{GeV}$ ), and for the following normalised effective couplings squared:

$$
\left(\frac{g_{h_{k} g g}^{\text {model }}}{g_{H g g}^{\mathrm{SM}}}\right)^{2},\left(\frac{g_{h_{k} \gamma \gamma}^{\text {model }}}{g_{H \gamma \gamma}^{\mathrm{SMM}}}\right)^{2},\left(\frac{g_{h_{k} Z Z}^{\text {model }}}{g_{H Z Z}^{\mathrm{SM}}}\right)^{2},\left(\frac{g_{h_{k} W W}^{\text {model }}}{g_{H W W}^{\mathrm{SM}}}\right)^{2},\left(\frac{g_{h_{k} f \bar{f}, \text { eff }}^{\text {model }}}{g_{H f \bar{f}}^{\mathrm{SM}}}\right)^{2},
$$

with $k \in\left\{1, \ldots, n_{\text {Higgs }}\right\}$ and $f \in\{b, \tau\}$. The reference coupling constants $g_{H Z Z}^{\mathrm{SM}}$ and $g_{H f \bar{f}}^{\mathrm{SM}}$ are defined in Eqs. (26) and (27), respectively, and $g_{H W W}^{\mathrm{SM}}$ by

$$
\left(g_{H W W}^{\mathrm{SM}}\right)^{2}=\left(\frac{e}{s_{\mathrm{W}}} m_{W}\right)^{2} .
$$

A convenient way to evaluate the ratios of the loop-induced effective couplings might be to use the ratio of partial decay widths:

$$
\left(\frac{g_{h_{k} v v^{\prime}}^{\text {model }}}{g_{H v v^{\prime}}^{\mathrm{SM}}}\right)^{2}=\left.\frac{\Gamma_{h_{k} \rightarrow v v^{\prime}}^{\text {model }}\left(m_{h_{k}}\right)}{\Gamma_{H \rightarrow v v^{\prime}}^{\mathrm{SM}}\left(m_{H}\right)}\right|_{m_{H}=m_{h_{k}}}
$$

with $v v^{\prime} \in\{g g, \gamma \gamma\}$. Of course, the user may apply Eq. (48) also for $v v^{\prime} \in$ $\{Z Z, W W\}$ if $m_{H}$ is large enough or the $\Gamma$ s for off-shell vector bosons are used. If the user has only the absolute partial decay widths, $\Gamma_{h_{k} \rightarrow F}^{\text {model }}\left(m_{h_{k}}\right)$, available, he or she can use the built-in functions for the calculation of SM quantities (see Table 12 below for details) in order to calculate the right-hand side of Eq. (48). 
From the input specified in Eqs. (24) and (46) HiggsBounds evaluates all partonic cross section ratios, as required in the Tevatron part of Input Option part, using the following formulae which are valid in the effective coupling approximation:

$$
\begin{array}{r}
\frac{\hat{\sigma}_{\text {model }}\left(g g \rightarrow h_{k}\right)}{\hat{\sigma}_{\mathrm{SM}}(g g \rightarrow H)}=\left(\frac{g_{h_{k} g g}^{\text {model }}}{g_{H g g}^{\mathrm{SM}}}\right)^{2}, \\
\frac{\hat{\sigma}_{\text {model }}\left(b \bar{b} \rightarrow h_{k}\right)}{\hat{\sigma}_{\mathrm{SM}}(b \bar{b} \rightarrow H)}=\frac{\hat{\sigma}_{\text {model }}\left(b g \rightarrow h_{k} b\right)}{\hat{\sigma}_{\mathrm{SM}}(b g \rightarrow H b)}=\left(\frac{g_{h_{k} \text { model }}^{\text {moff }}}{g_{H b \bar{b}}^{\mathrm{SM}}}\right)^{2}, \\
\frac{\hat{\sigma}_{\text {model }}\left(q \bar{q}^{\prime} \rightarrow h_{k} W^{+}\right)}{\hat{\sigma}_{\mathrm{SM}}\left(q \bar{q}^{\prime} \rightarrow H W^{+}\right)}=\frac{\hat{\sigma}_{\text {model }}\left(q^{\prime} \bar{q} \rightarrow h_{k} W^{-}\right)}{\hat{\sigma}_{\mathrm{SM}}\left(q^{\prime} \bar{q} \rightarrow H W^{-}\right)}=\left(\frac{g_{h_{k} W W}^{\text {model }}}{g_{H W W}^{\mathrm{SM}}}\right)^{2}, \\
\frac{\hat{\sigma}_{\text {model }}\left(q^{\prime \prime} \bar{q}^{\prime \prime} \rightarrow h_{k} Z\right)}{\hat{\sigma}_{\mathrm{SM}}\left(q^{\prime \prime} \bar{q}^{\prime \prime} \rightarrow H Z\right)}=\left(\frac{g_{h_{k} Z Z}^{\text {model }}}{g_{H Z Z}^{\mathrm{SM}}}\right)^{2},
\end{array}
$$

for $\left(q, q^{\prime}\right) \in\{(u, d),(c, s)\}$ and $q^{\prime \prime} \in\{u, d, c, s, b\}$.

In order to facilitate the calculation of the ratios of the hadronic cross sections for $h_{k}$ production via VBF, we obtained the following SM cross section ratio for $p \bar{p}$ collisions with $1.96 \mathrm{TeV}$ centre-of-mass energy using VBFNLO [29]:

$$
\frac{\sigma_{\mathrm{SM}}(p \bar{p} \rightarrow H \text { via } W W \text { fusion })}{\sigma_{\mathrm{SM}}(p \bar{p} \rightarrow H \text { via } Z Z \text { fusion })} \approx 3.35
$$

The right-hand side is a mean value. The actual value varies slightly with $m_{H}$ $\left( \pm 3.6 \%\right.$ in the relevant mass range of $\left.70 \mathrm{GeV}<m_{H}<300 \mathrm{GeV}\right)$. Using this mean and neglecting interference effects, which affect the result below $1 \%$ (cf. [53]), we can calculate the proportions of $W W$ - and $Z Z$-fusion contributions to the SM VBF Higgs production cross section for $p \bar{p}$ collisions with $1.96 \mathrm{TeV}$ centre-of-mass energy:

$$
\begin{aligned}
R_{\mathrm{VBF}}^{W W} & :=\frac{\sigma_{\mathrm{SM}}(p \bar{p} \rightarrow H \text { via } W W \text { fusion })}{\sigma_{\mathrm{SM}}(p \bar{p} \rightarrow H \text { via } \mathrm{VBF})}=77 \%, \\
R_{\mathrm{VBF}}^{Z Z} & :=\frac{\sigma_{\mathrm{SM}}(p \bar{p} \rightarrow H \text { via } Z Z \text { fusion })}{\sigma_{\mathrm{SM}}(p \bar{p} \rightarrow H \text { via } \mathrm{VBF})}=23 \% .
\end{aligned}
$$

The slight variation of the right-hand side of Eq. (53) with $m_{H}$ causes a variation of the above proportions by less than $1 \%$.

Using the above SM predictions, we can calculate the model predictions for the ratios of the hadronic cross sections for $h_{k}$ production via VBF quite accurately, as follows:

$$
\frac{\sigma_{\text {model }}\left(p \bar{p} \rightarrow h_{k} \text { via } \mathrm{VBF}\right)}{\sigma_{\mathrm{SM}}(p \bar{p} \rightarrow H \text { via } \mathrm{VBF})}=R_{\mathrm{VBF}}^{W W}\left(\frac{g_{h_{k} W W}^{\text {model }}}{g_{H W W}^{\mathrm{SM}}}\right)^{2}+R_{\mathrm{VBF}}^{Z Z}\left(\frac{g_{h_{k} Z Z}^{\text {model }}}{g_{H Z Z}^{\mathrm{SM}}}\right)^{2} .
$$


Branching ratios, additional to the ones we have already described when discussing the LEP limits in Section 2.2.2, are evaluated along the lines of Eq. (33):

$$
\begin{aligned}
\operatorname{BR}_{\text {model }}\left(h_{k} \rightarrow \gamma \gamma\right) & =\left.\mathrm{BR}_{\mathrm{SM}}(H \rightarrow \gamma \gamma)\left(m_{H}\right) \frac{\Gamma_{\text {tot }}^{\mathrm{SM}}\left(m_{H}\right)}{\Gamma_{\text {tot }}\left(h_{k}\right)}\right|_{m_{H}=m_{h_{k}}} \times \\
& \times\left(\frac{g_{h_{k} \gamma \gamma}^{\text {model }}}{g_{H \gamma \gamma}^{\mathrm{SM}}}\right)^{2}, \\
\operatorname{BR}_{\text {model }}\left(h_{k} \rightarrow W W\right) & =\left.\mathrm{BR}_{\mathrm{SM}}(H \rightarrow W W)\left(m_{H}\right) \frac{\Gamma_{\text {tot }}^{\mathrm{SM}}\left(m_{H}\right)}{\Gamma_{\text {tot }}\left(h_{k}\right)}\right|_{m_{H}=m_{h_{k}}} \times \\
& \times\left(\frac{g_{h_{k} W W}^{\text {model }}}{g_{H W W}^{\mathrm{SM}}}\right)^{2} .
\end{aligned}
$$

At this point, all quantities which are required as Tevatron input by Input Option part are evaluated using the effective coupling approximation. The evaluation of the model predictions $Q_{\text {model }}(X)$ can then proceed as exemplified in Section 2.3.2 above.

For search topologies, of which the amplitude is proportional to one monomial of Higgs coupling constants, effective couplings just rescale all kinematical distributions of the reference process by a common factor without changing their shape. Consequently, in such cases, limits on topological cross sections can be exactly applied. For the application of limits obtained from SM analyses, which combine a set of search topologies, the SM-likeness check (see Section 2.1) ensures that the kinematical distributions arising from models that pass this check closely resemble the distributions of the SM. Thus, also in this case, limits on topological cross sections can be exactly applied.

Two slightly less straight-forward cases require further discussion.

a) For the single Higgs production topology, we add the gluon-fusion and $b \bar{b}$ annihilation contribution together. Firstly, one might expect that the boost distribution of the produced Higgs boson is different for the two partonic processes and thus the kinematical distributions of the Higgs decay products would depend on the relative contributions of the two processes to the signal. However, it turns out that the gluon and bottom parton distributions (using MSTW 2008 PDFs [54]) in the relevant region (lower $x$ values) are, to a very good approximation, proportional to each other, i.e. the two parton processes do not give rise to different boost distributions of the produced Higgs boson. Secondly, it is known that the event selection cuts used in single Higgs analyses select Higgs bosons with a certain $p_{T}$, which is known to lead to different distributions for processes arising from gluon-gluon and $b \bar{b}$ initial states. This change in the kinematical distribution becomes relevant if the coupling of the Higgs to a bottom quark is strongly enhanced such that the $b \bar{b}$ annihilation 
competes with gluon-fusion contribution, where the latter dominates in the SM. Indeed, it turns out that, for such a scenario, out of the implemented set of analyses, the most sensitive are those that involve the search topology $p \bar{p} \rightarrow H \rightarrow \tau \tau$ [24]25]. However, these analyses reject events with extra jets of large transverse momentum, where the deviation of the $p_{T}$-distributions becomes substantial [55]. Therefore, we do not expect a strong influence of the moderately changed kinematical distributions on the cross section limits. This expectation is supported by the fact that we reproduce the MSSM exclusion plots in [24,25] reasonably well.

b) For Higgs production via vector boson fusion, a deviation from the SM proportion of $W W$ and $Z Z$ initiated processes caused by different effective couplings $g_{h_{k} Z Z}^{2}$ and $g_{h_{k} W W}^{2}$, does not lead to significant changes in the kinematical distributions compared to the SM reference process.

In summary, for the implemented set of analyses, we expect the limits on topological cross sections to be applicable with high accuracy for models where the Higgs sector can be faithfully parametrised with effective couplings.

\section{$3 \quad$ HiggsBounds Operating Instructions}

There are three formats in which the program HiggsBounds can be used:

- Library of subroutines

- Command-line version

- Online version

The most widely applicable format of HiggsBounds is the command-line version, since this reads all the model data from text files and thus this model data can be generated using any package the user wishes. The library of HiggsBounds subroutines allows HiggsBounds to be called within other programs. If the user just wishes to check a few parameter points, the online version provides quick access to all the functionality of HiggsBounds, without the need to install the code.

The HiggsBounds code, the online version and documentation can all be found at the URL www.ippp.dur.ac.uk/HiggsBounds .

The HiggsBounds code is provided in either Fortran 77 or Fortran 90. Both codes provide exactly the same functionality and have exactly the same operating instructions. In fact, the Fortran 77 and Fortran 90 versions of the HiggsBounds subroutines can even be called within codes written in Fortran 90 and Fortran 77, respectively. Both codes have also been tested with a variety of Fortran compilers, including the free gnu compilers which accompany 
most Linux distributions. Therefore, the user may download either code and the difference will only be apparent if the user wishes to examine the structure of the code 6 .

The library of subroutines, the command-line version and the online version share a common set of features, which we will describe first. We will then give operating instructions for each of these three HiggsBounds formats individually.

\subsection{Common features: Input}

HiggsBounds requires four types of input:

- the number of neutral Higgs bosons in the model under study (nH)

- the set of experimental analyses which should be considered (whichexpt)

- the theoretical predictions of the model under study (a set of input arrays)

- the format of these theoretical predictions (whichinput)

Table 4 contains further information on the variable $\mathrm{nH}$, and the possible values of whichexpt are described in Table 5. Note that the option whichexpt='singH' should only be used if neither processes involving Higgs pair production at LEP nor processes involving the $h_{j} \rightarrow h_{i} h_{i}$ decay are relevant. However, if these conditions are met, this option can save significantly on computing time.

HiggsBounds expects the theoretical input to be in one of three formats, labelled by the variable whichinput. These formats were described in detail in Section 2 and are briefly summarised in Table 6. In Table 7 and Table 8 we assign names to all of the possible input arrays (each array is defined in terms of the notation used in Section 2). These names will prove useful when we describe the input requirements of each version of HiggsBounds individually.

\begin{tabular}{ll}
$\mathrm{nH}$ & (integer) \\
\hline $1-9$ & $\begin{array}{l}\text { By default, HiggsBounds can be applied to models with } \\
\text { between } 1 \text { and } 9 \text { neutral Higgs bosons. }\end{array}$ \\
& (This range can easily be extended by the user if required.)
\end{tabular}

Table 4

The possible values of the variable $\mathrm{nH}$, which labels the number of neutral Higgs bosons in the model under study.

6 The maintenance of two separate codes is primarily intended to provide an efficient way for the authors to confirm that each update is free from implementation errors. 


\begin{tabular}{ll} 
whichexpt & $($ character $($ LEN=5) $)$ \\
\hline LandT & both LEP and Tevatron analyses \\
onlyL & only LEP analyses \\
onlyT & only Tevatron analyses \\
singH & only analyses for processes involving one Higgs boson
\end{tabular}

Table 5

The possible values of the variable whichexpt, which indicates which subset of experimental analyses will be considered by HiggsBounds.

\begin{tabular}{|c|c|}
\hline $\operatorname{effC}$ & $\begin{array}{l}\text { Masses, total decay widths, } \\
\text { ratios of effective couplings squared, } \mathrm{BR}\left(h_{j} \rightarrow h_{i} h_{i}\right) \text {. }\end{array}$ \\
\hline part & $\begin{array}{l}\text { Masses, total decay widths, ratios of LEP cross sections, } \\
\text { mainly ratios of partonic Tevatron cross sections, branching ratios. }\end{array}$ \\
\hline hadr & $\begin{array}{l}\text { Masses, total decay widths, ratios of LEP cross sections, } \\
\text { ratios of hadronic Tevatron cross sections, branching ratios. }\end{array}$ \\
\hline
\end{tabular}

Table 6

The possible values of the variable whichinput, which indicates the format of the theoretical predictions provided by the user. (See Section 2 for a more detailed description of each of these settings). 


\begin{tabular}{|c|c|c|c|}
\hline input arrays & \multicolumn{3}{|c|}{ (double precision) } \\
\hline $\operatorname{Mh}(\mathrm{nH})$ & $m_{h_{i}}$ & in $\mathrm{GeV}$ & \\
\hline GammaTotal (nH) & $\Gamma_{\text {tot }}\left(h_{i}\right)$ & in $\mathrm{GeV}$ & \\
\hline g2hjbb (nH) & $\left(\frac{g_{h_{j}(\mathrm{OP})}^{\text {model }}}{g_{H(\mathrm{OP})}^{\mathrm{SM}}}\right)^{2}$ & $\mathrm{OP}=$ & $b \bar{b}$ \\
\hline g2hjtautau (nH) & & & $\tau^{+} \tau^{-}$ \\
\hline g2hjWW (nH) & & & $W W$ \\
\hline g2hjZZ (nH) & & & $Z Z$ \\
\hline g2hjgaga (nH) & & & $\gamma \gamma$ \\
\hline g2hjgg (nH) & & & $g g$ \\
\hline g2hjhiZ (nH,nH) & $\left(\frac{g_{h_{h} h_{i} Z}^{\text {model }}}{g_{H H^{\prime} Z}^{\text {ref }}}\right)^{2}$ & & \\
\hline $\mathrm{BR} \_\mathrm{hjbb}(\mathrm{nH})$ & $\mathrm{BR}\left(h_{j} \rightarrow \mathrm{OP}\right)$ & $\mathrm{OP}=$ & $b \bar{b}$ \\
\hline BR_hjtautau (nH) & & & $\tau^{+} \tau^{-}$ \\
\hline $\mathrm{BR} h \mathrm{~h} \mathrm{WW}(\mathrm{nH})$ & & & $W W$ \\
\hline BR_hjgaga $(\mathrm{nH})$ & & & $\gamma \gamma$ \\
\hline BR_hjhihi $(\mathrm{nH}, \mathrm{nH})$ & $\mathrm{BR}\left(h_{j} \rightarrow h_{i} h_{i}\right)$ & & \\
\hline
\end{tabular}

Table 7

Input arrays for model predictions for effective normalised squared couplings and branching ratios recognised by HiggsBounds. The size of each array is given in brackets in the first column. See Section 2 for the description of the notation used in the second column. The elements of BR hjhihi are ordered such that $\operatorname{BR\_ hjhihi}(j, i)=B R\left(h_{j} \rightarrow h_{i} h_{i}\right)$. 


\begin{tabular}{|c|c|c|c|}
\hline \multirow{2}{*}{$\frac{\text { input arrays cont. }}{\text { CS_lep_hjZ_ratio(nH) }}$} & \multicolumn{3}{|c|}{ (double precision) } \\
\hline & $R_{\sigma}(P)$ & $P=$ & $e^{+} e^{-} \rightarrow h_{j} Z$ \\
\hline CS_lep_hjhi_ratio $(\mathrm{nH}, \mathrm{nH})$ & & & $e^{+} e^{-} \rightarrow h_{j} h_{i}$ \\
\hline CS_tev_pp_hj_ratio(nH) & & & $p \bar{p} \rightarrow h_{j}$ \\
\hline CS_tev_pp_hjb_ratio(nH) & & & $p \bar{p} \rightarrow b h_{j}$ \\
\hline CS_tev_pp_hjW_ratio(nH) & & & $p \bar{p} \rightarrow h_{j} W$ \\
\hline CS_tev_pp_hjZ_ratio (nH) & & & $p \bar{p} \rightarrow h_{j} Z$ \\
\hline CS_tev_pp_vbf_ratio(nH) & & & $p \bar{p} \rightarrow h_{j}$ via $\mathrm{VBF}$ \\
\hline CS_tev_gg_hj_ratio(nH) & $R_{n m}^{h_{j}}$ & $n m=$ & $g g$ \\
\hline CS_tev_bb_hj_ratio(nH) & & & $b \bar{b}$ \\
\hline CS_tev_ud_hjWp_ratio(nH) & $R_{n m}^{h_{j}+W^{+}}$ & $n m=$ & $u \bar{d}$ \\
\hline CS_tev_cs_hjWp_ratio(nH) & & & $c \bar{s}$ \\
\hline CS_tev_ud_hjWm_ratio(nH) & $R_{n m}^{h_{j}+W^{-}}$ & $n m=$ & $d \bar{u}$ \\
\hline CS_tev_cs_hjWm_ratio(nH) & & & $s \bar{c}$ \\
\hline CS_tev_dd_hjZ_ratio(nH) & $R_{n m}^{h_{j}+Z}$ & $n m=$ & $d \bar{d}$ \\
\hline CS_tev_uu_hjZ_ratio (nH) & & & $u \bar{u}$ \\
\hline CS_tev_ss_hjZ_ratio (nH) & & & $s \bar{s}$ \\
\hline CS_tev_cc_hjZ_ratio (nH) & & & $c \bar{c}$ \\
\hline CS_tev_bb_hjZ_ratio (nH) & & & $b \bar{b}$ \\
\hline CS_tev_bg_hjb_ratio(nH) & $R_{n m}^{h_{j}+b}$ & $n m=$ & $b g, \bar{b} g$ \\
\hline
\end{tabular}

Table 8

Input arrays for model predictions for cross section ratios recognised by HiggsBounds. The size of each array is given in brackets in the first column. The LEP or hadronic Tevatron cross section ratios $R_{\sigma}(P)$ are defined in Eq. (43) and the partonic Tevatron cross section ratios $R_{n m}^{h_{j}+y}$ are defined in Eq. (44)). 


\subsection{Common features: Output}

HiggsBounds provides the user with four types of output:

- whether the parameter point is excluded at the 95\% C.L. or not (HBresult)

- the identifying number of the process with the highest statistical sensitivity (chan).

- the number of Higgs bosons which have contributed to the theoretical rate for this process (ncombined)

- the ratio of the theoretical rate $Q_{\text {model }}$ to the observed limit $Q_{\text {obs }}$ for this process (obsratio).

Table 9 shows the possible values of HBresult and obsratio, which are complementary. Tables 10 and 11 discuss chan and ncombined respectively. If the library of subroutines or the command-line versions are used, the key to the process numbers is written in the file Key.dat. In the online version, this information appears on the screen.

\begin{tabular}{lll}
\hline $\begin{array}{l}\text { HBresult } \\
\text { (integer) }\end{array}$ & $\begin{array}{l}\text { obsratio } \\
\text { (double precision) }\end{array}$ \\
\hline 0 & $\geq 1.0$ & parameter point is excluded \\
1 & $<1.0$ & parameter point is not excluded \\
-1 & $\leq 0.0$ & invalid parameter set
\end{tabular}

Table 9

The possible values of the output variables HBresult and obsratio, which indicate whether a parameter point has been excluded at the 95\% C.L. by the experimental results under consideration.

\begin{tabular}{ll} 
chan & (integer) \\
\hline 1-[\# of considered analyses $]$ & $\begin{array}{l}\text { See the file Key.dat for the definition of each } \\
\text { process number. Key.dat is automatically } \\
\text { generated when either the command line or the } \\
\text { subroutine version of HiggsBounds are used. }\end{array}$
\end{tabular}

Table 10

Further information about the output variable chan, which stores the reference number of the process with the highest statistical sensitivity. 


\begin{tabular}{|c|c|}
\hline $1-n_{H}$ & $\begin{array}{l}\text { Number of Higgs bosons which have contributed to the theoretical } \\
\text { rate for this process. The number depends on delta_Mh_LEP } \\
\text { or delta_Mh_TEV. }\end{array}$ \\
\hline
\end{tabular}

Table 11

Further information about the output variable ncombined.

\subsection{Library of subroutines}

\section{Installation}

The HiggsBounds code can be compiled to form a library of subroutines using the following commands:

./configure

make libHB

A program which wishes to use the HiggsBounds subroutines can be compiled and linked to the library by adding $-\mathrm{L}<\mathrm{HBpath}>-1 \mathrm{HB}$ to the command line, for example,

gfortran myprog.f90 -o myprog -L<HBpath> -IHB

where <HBpath> is the location of the HiggsBounds library.

\section{Subroutine initialize_HiggsBounds}

The subroutine initialize_HiggsBounds must be called before any other HiggsBounds subroutine. It performs some preparatory operations such as reading in the tables of data. It is called as:

call initialize_HiggsBounds(nH, whichexpt)

When using the HiggsBounds subroutines in another code, the subroutine initialize_HiggsBounds must be called only once, before any other HiggsBounds subroutine is called.

Subroutines run_HiggsBounds_effC, run_HiggsBounds_part and run_HiggsBounds_hadr

The subroutines run_HiggsBounds_effC, run_HiggsBounds_part and run_HiggsBounds_hadr perform the main part of the HiggsBounds calculations. They set the value of whichinput to be effC, part and hadr respectively and therefore require different arguments. These subroutines are called 
call run_HiggsBounds_effC (nH, Mh, GammaTotal,

\& g2hjbb,g2hjtautau,g2hjWW,g2hjZZ,

\& g2hjgaga,g2hjgg,g2hjhiZ,

\& BR_hjhihi,

\& HBresult, chan,

\& obsratio, ncombined

call run_HiggsBounds_part ( $\mathrm{nH}, \mathrm{Mh}$,

$\begin{array}{ll}\& & \text { CS_lep_hjZ_ratio, CS_lep_hjhi_ratio, } \\ \& & \text { CS_tev_gg_hj_ratio, CS_tev_bb_hj_ratio, } \\ \& & \text { CS_tev_bg_hjb_ratio, } \\ \& & \text { CS_tev_ud_hjWp_ratio, CS_tev_cs_hjWp_ratio, } \\ \& & \text { CS_tev_ud_hjWm_ratio, CS_tev_cs_hjWm_ratio, } \\ \& & \text { CS_tev_dd_hjZ_ratio, CS_tev_uu_hjZ_ratio, } \\ \& & \text { CS_tev_ss_hjZ_ratio, CS_tev_cc_hjZ_ratio, } \\ \& & \text { CS_tev_bb_hjZ_ratio, } \\ \& & \text { CS_tev_pp_vbf_ratio, } \\ \& & \text { BR_hjbb,BR_hjtautau, } \\ \& & \text { BR_hjWW,BR_hjgaga, } \\ \& & \text { BR_hjhihi, } \\ \& & \text { HBresult, chan, } \\ \& & \text { obsratio, ncombined }\end{array}$

call run_HiggsBounds_hadr(nH, Mh,

$\begin{array}{ll}\& & \text { CS_lep_hjZ_ratio, } \\ \& & \text { CS_tev_pp_hj_ratio, CS_tev_pp_hjb_ratio, } \\ \& & \text { CS_tev_pp_hjW_ratio, CS_tev_pp_hjZ_ratio, } \\ \& & \text { CS_tev_pp_vbf_ratio, } \\ \& & \text { BR_hjbb,BR_hjtautau, } \\ \& & \text { BR_hjWW,BR_hjgaga, } \\ \& & \text { BR_hjhihi, } \\ \& & \text { HBresult, chan, } \\ \& & \text { obsratio, ncombined }\end{array}$

Each of these arguments must be supplied. However, if a branching ratio, effective coupling or cross section is believed to be irrelevant, the corresponding array may be filled with zeros. This will ensure that the value of $Q_{\text {model }}$ for processes involving this quantity will also be zero. For example, in the MSSM, processes involving the decay $h_{j} \rightarrow \gamma \gamma$ will rarely be the process with the highest statistical sensitivity of $Q_{\text {model }} / Q_{\text {expec }}$ and, consequently, it may be convenient to set the arrays g2hjgaga and BR_hjgaga to zero for simplicity. 
Also, depending on the value given for whichexpt, some of the input arrays will be ignored within HiggsBounds. For example, if whichexpt='onlyT', the branching ratio for the Higgs cascade decay $h_{j} \rightarrow h_{i} h_{i}$ will not be relevant. Therefore, setting this array to zero in this case will not affect the HiggsBounds results in this case.

\section{Subroutine finish_HiggsBounds}

The subroutine finish_HiggsBounds should be called once at the end of the program, after all other HiggsBounds subroutines. It is called as:

call finish_HiggsBounds

Functions for Standard Model branching ratios, total decay width and cross sections

The HiggsBounds library also allows users access to the Standard Model Higgs branching ratios, total decay width and production cross sections, which are used internally by HiggsBounds. We use Standard Model Higgs branching ratios and total decay width from the program HDECAY 3.303 [26]. The SM hadronic cross sections have been obtained from the TEV4LHC Higgs Working Group [27] (see Table12 for references to the original works) with the exception of the $\sigma^{\mathrm{SM}}(p \bar{p} \rightarrow b g \rightarrow b H)$ cross section. The latter cross section has been calculated with the program HJET 1.1 [28] for a set of different cuts on the transverse momentum and pseudo-rapidity of the $b$-quark, which are needed internally in order to apply correctly the results of some Tevatron analyses and which were not available from [27]. From this set, only the cross section without cuts is externally provided.

\section{Examples}

We have provided three example programs which demonstrate the use of the HiggsBounds subroutines. The first example relates to the Fourth Generation Model and is contained in the file example-SM_vs_4thGen.F (see Fig. 4 below for a code listing). This program uses the HiggsBounds functions for the SM branching ratios and SM total decay width to calculate the Higgs decay width and the effective normalised squared couplings in the SM and a simple Fourth Generation Model. This information is then used as input for the subroutine run_HiggsBounds_effC, which is called once with SM input and once with Fourth Generation Model input. The example will be described in more detail in Section 4.2. Once the HiggsBounds library has been compiled (using ./configure ; make libHB as described previously), the code

\footnotetext{
7 In the Fortran 90 version of the code, the subroutine finish_HiggsBounds is used to deallocate the allocatable arrays used within HiggsBounds.
} 


\begin{tabular}{|c|c|c|c|c|}
\hline function & (double precisic & & & \\
\hline SMGamma_h (Mh) & $\Gamma_{\text {tot }}^{\mathrm{SM}}\left(h_{i}\right)$ & & & 26 \\
\hline SMBR_Hbb (Mh) & $\mathrm{BR}^{\mathrm{SM}}(H \rightarrow \mathrm{OP})$ & $\mathrm{OP}=$ & $b \bar{b}$ & 26 \\
\hline SMBR_Htautau (Mh) & & & $\tau^{-} \tau^{+}$ & 26 \\
\hline SMBR_HWW (Mh) & & & $W W$ & [26] \\
\hline SMBR_Hgamgam (Mh) & & & $\gamma \gamma$ & 26 \\
\hline SMBR_Hgg (Mh) & & & $g g$ & [26] \\
\hline SMBR_HZgam (Mh) & & & $Z \gamma$ & 26 \\
\hline SMBR_HZZ (Mh) & & & $Z Z$ & [26] \\
\hline SMBR_Htoptop (Mh) & & & $t \bar{t}$ & [26] \\
\hline $\operatorname{SMBR} H \mathrm{Hcc}(\mathrm{Mh})$ & & & $c \bar{c}$ & [26] \\
\hline SMBR_Hss (Mh) & & & $s \bar{s}$ & [26] \\
\hline SMBR_Hmumu (Mh) & & & $\mu^{-} \mu^{+}$ & 26 \\
\hline SMCS_tev_pp_qq_HW (Mh) & $\sigma^{\mathrm{SM}}(P)$ & $\mathrm{P}=$ & $p \bar{p} \rightarrow q \bar{q} \rightarrow H W$ & 56 \\
\hline SMCS_tev_pp_qq_HZ (Mh) & & & $p \bar{p} \rightarrow q \bar{q} \rightarrow H Z$ & 56 \\
\hline SMCS_tev_pp_gg_H(Mh) & & & $p \bar{p} \rightarrow g g \rightarrow H$ & 50,51 \\
\hline SMCS_tev_pp_bb_H(Mh) & & & $p \bar{p} \rightarrow b \bar{b} \rightarrow H$ & 52 \\
\hline SMCS_tev_pp_vbf_H (Mh) & & & $p \bar{p} \rightarrow H$ via $\mathrm{VBF}$ & 57 \\
\hline SMCS_tev_pp_bg_Hb (Mh) & & & $p \bar{p} \rightarrow b g \rightarrow H b$ & 28 \\
\hline
\end{tabular}

Table 12

Standard Model branching ratios, total decay widths in units of $\mathrm{GeV}$ and hadronic Tevatron cross sections in units of pikobarn provided as functions by HiggsBounds, together with references. Each function takes a Higgs mass Mh (double precision) as its argument.

example-SM_vs_4thGen.F can be compiled and run with the commands:

gfortran example-SM_vs_4thGen.F -o HBfourthgen -L<HBpath> - lHB ./HBfourthgen

where $<$ HBpath $>$ is the location of the HiggsBounds library.

The files HBwithFH.F and HBwithCPsuperH.f demonstrate the use of HiggsBounds subroutines with the publically available programs FeynHiggs [14] and $\mathrm{CPsuperH}$ [18], respectively. We refer the reader to the extensive comments contained within these example files for further details. 


\subsection{Command line version}

\section{Installation}

In order to be able to call HiggsBounds from the command line, it should be compiled using the commands

./configure

make

Command line and input file format

In the command-line usage of HiggsBounds, the arrays containing the theoretical model predictions are read from text files. The other options are specified in the command line, which is of the form:

\section{./HiggsBounds <whichexpt> <whichinput> <nH> <prefix>}

The variable <prefix $>$ is a string which is added to the front of input and output file names and may include directory names or other identifying information.

Table 13 describes the contents of each input file. Note that each input file should start with a line number. The input files should not contain any comments or blank lines. The line number identifies the predictions which belong to the same model parameter point in different files.

Care should be taken with the order of the array elements in the files. This is best illustrated by an example, where we will use $n_{H}=3$. The one dimensional arrays, e.g. Mh, should be given in the order

$\operatorname{Mh}(1), \operatorname{Mh}(2), \operatorname{Mh}(3)$

However, not all of the elements of the two dimensional arrays are required. Only the lower left triangle (including the diagonal) is required from the arrays g2hjhiz and lepCS_hjhi_ratio, since they are symmetric, e.g.

$$
\left(\begin{array}{lll}
\operatorname{ghhhiZ}(1,1) & g 2 h j h i Z(1,2) & \operatorname{g} 2 h j h i Z(1,3) \\
\operatorname{g} 2 h j h i Z(2,1) & g 2 h j h i Z(2,2) & g 2 h j h i Z(2,3) \\
\operatorname{g} 2 h j h i Z(3,1) & g 2 h j h i Z(3,2) & g 2 h j h i Z(3,3)
\end{array}\right)
$$

i.e. the elements in the input file should be written in the order

$$
\begin{aligned}
& \operatorname{g} 2 \operatorname{hhiZ}(1,1), \operatorname{g} 2 \operatorname{hjhiZ}(2,1), \operatorname{g} 2 \operatorname{hjhiZ}(2,2), \operatorname{g} 2 h j h i Z(3,1), \\
& \operatorname{g} 2 \operatorname{hhiZ}(3,2), \operatorname{g} 2 \operatorname{hjhiZ}(3,3) .
\end{aligned}
$$




\begin{tabular}{|c|c|}
\hline file name & data format \\
\hline MH_GammaTot.dat & k, Mh, GammaTotal \\
\hline effC.dat & $\begin{array}{l}\text { k, g2hjbb,g2hjtautau,g2hjWW,g2hjZZ, } \\
\text { g2hjgaga,g2hjgg, } \\
\text { some elements of g2hjhiZ } \\
\text { (lower left triangle - see example) }\end{array}$ \\
\hline LEP_HZ_CS_ratios.dat & k, CS_lep_hjZ_ratio \\
\hline LEP_2H_CS_ratios.dat & $\begin{array}{l}\text { some elements of CS_lep_hjhi_ratio } \\
\text { (lower left triangle - see example) }\end{array}$ \\
\hline TEV_H_0jet_partCS_ratios.dat & k, CS_tev_gg_hj_ratio,CS_tev_bb_hj_ratio \\
\hline TEV_H_1jet_partCS_ratios.dat & k CS_tev_bb_hjb_ratio \\
\hline TEV_HW_partCS_ratios.dat & $\begin{array}{l}\text { k, CS_tev_ud_hjWp_ratio, } \\
\text { CS_tev_cs_hjWp_ratio, } \\
\text { CS_tev_ud_hjWm_ratio, } \\
\text { CS_tev_cs_hjWm_ratio }\end{array}$ \\
\hline TEV_HZ_partCS_ratios.dat & $\begin{array}{l}\text { k, CS_tev_dd_hjZ_ratio, } \\
\text { CS_tev_uu_hjZ_ratio, } \\
\text { CS_tev_ss_hjZ_ratio, } \\
\text { CS_tev_cc_hjZ_ratio, } \\
\text { CS_tev_bb_hjZ_ratio }\end{array}$ \\
\hline TEV_H_vbf_hadCS_ratios.dat & k, CS_tev_pp_vbf_ratio \\
\hline TEV_1H_hadCS_ratios.dat & $\begin{array}{l}\text { k, CS_tev_pp_hj_ratio, } \\
\text { CS_tev_pp_hjb_ratio, } \\
\text { CS_tev_pp_hjW_ratio, } \\
\text { CS_tev_pp_hjZ_ratio, } \\
\text { CS_tev_pp_vbf_ratio }\end{array}$ \\
\hline BR_1H.dat & k, BR_hjbb,BR_hjtautau,BR_hjWW,BR_hjgaga \\
\hline BR_2H.dat & $\begin{array}{l}\text { k, some elements of BR_hjhihi } \\
\text { (row by row, without diagonal } \\
\text { - see example) }\end{array}$ \\
\hline additional.dat(optional) & $\mathrm{k}, \ldots$ \\
\hline
\end{tabular}

Table 13

Names and data format of all HiggsBounds input files. The right column shows the order of the input data arrays within one line of the input file. For the order within the arrays, see example. $\mathrm{k}$ is the line number. Note that the array CS_tev_pp_vbf_ratio appears in two different input files. However, these files will never be required by HiggsBounds simultaneously. 
For the array BR_hjhihi, only the off-diagonal components are required

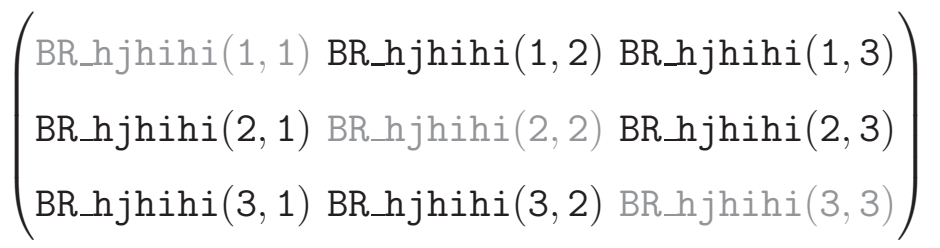

since the diagonal elements are not physical quantities. Therefore, the elements should be written in the order

$$
\begin{aligned}
& \text { BR_hjhihi (1,2), BR_hjhihi (1,3), BR_hjhihi }(2,1) \text {, BR_hjhihi }(2,3) \text {, } \\
& \text { BR_hjhihi }(3,1) \text {, BR_hjhihi }(3,2)
\end{aligned}
$$

in the input file.

The file additional. dat is optional. If it is included, it can have any number of columns greater than 1 (as for the previous files, the first entry on each line should be the line number). It is envisaged that this input file will be particularly useful when parameter scans are performed over a variable which is not required by HiggsBounds but helpful plotting the results. For example, in the case of the complex MSSM, additional. dat could be used to store the values of $\tan \beta$ and the charged Higgs mass.

As in the subroutine version, the command line version of HiggsBounds expects a subset of the total list of input arrays, which depends on the chosen setting of whichinput. The maximal list of files used for each value of whichinput is given in Table 5 .

As discussed for the subroutine version, some of the arrays will not be relevant for some of the choices for whichexpt. The command line version of HiggsBounds will consider the list of input files appropriate to the setting whichinput and then only attempt to read any of these input files if the value chosen for whichexpt means that at least one of the arrays it contains will be directly used. Table 15 contains a list of which input files are actually relevant to each value of whichexpt. For example, if whichinput = 'hadr', and whichexpt $=$ 'LandT', then HiggsBounds requires the input files:

MH_GammaTot.dat, BR_2H.dat, BR_1H.dat, LEP_HZ_CS_ratios.dat, LEP_2H_CS_ratios.dat, TEV_1H_hadCS_ratios.dat .

However, if whichinput $=$ 'hadr' and whichexpt = 'onlyL', HiggsBounds requires the input files:

MH_GammaTot.dat, BR_2H.dat, BR_1H.dat, LEP_HZ_CS_ratios.dat, LEP_2H_CS_ratios.dat . 


\begin{tabular}{lll}
\hline whichinput = 'part', & 'hadr', & 'effC' \\
\hline MH_GammaTot.dat & MH_GammaTot.dat & MH_GammaTot.dat \\
BR_2H.dat & BR_2H.dat & effC.dat \\
BR_1H.dat & BR_1H.dat & BR_2H.dat \\
LEP_HZ_CS_ratios.dat & LEP_HZ_CS_ratios.dat & additional.dat \\
LEP_2H_CS_ratios.dat & LEP_2H_CS_ratios.dat & \\
TEV_H_0jet_partCS_ratios.dat & TEV_1H_hadCS_ratios.dat & \\
TEV_H_1jet_partCS_ratios.dat & additional.dat & \\
TEV_HW_partCS_ratios.dat & \\
TEV_HZ_partCS_ratios.dat & & \\
TEV_H_vbf_hadCS_ratios.dat & \\
additional.dat & & \\
\hline
\end{tabular}

Table 14

The list of possible input files for each value of whichinput. Note that some input files may not be relevant, depending on the value of whichexpt. In this case, they are not required. See Table 15 for more details.

As a third example, if whichinput = 'hadr' and whichexpt = 'onlyT', HiggsBounds requires the input files:

MH_GammaTot.dat, BR_1H.dat, TEV_1H_hadCS_ratios.dat .

In each of these three examples, HiggsBounds will also read the file

$$
\text { additional.dat }
$$

if it exists.

As for the subroutine version, if the user does not require processes involving a particular branching ratio or cross section ratio to be checked by HiggsBounds, that particular array can be filled with zeros.

\section{Output file format}

When the command line version of HiggsBounds is used, the output is written to the file <prefix>HiggsBounds_results.dat. A sample of the output is shown in Fig. 2. The key to the process numbering is written to <prefix>Key.dat. 


\begin{tabular}{|c|c|c|c|c|}
\hline \multirow[t]{2}{*}{ name of input file } & \multicolumn{4}{|c|}{$\begin{array}{l}\text { values of whichexpt } \\
\text { which this file is relevant to }\end{array}$} \\
\hline & LandT & onlyL & onlyT & $\operatorname{singH}$ \\
\hline MH_GammaTot.dat & $\mathrm{y}$ & $\mathrm{y}$ & $\mathrm{y}$ & $\mathrm{y}$ \\
\hline effC.dat & $\mathrm{y}$ & $\mathrm{y}$ & $\mathrm{y}$ & $\mathrm{y}$ \\
\hline LEP_HZ_CS_ratios.dat & $\mathrm{y}$ & $\mathrm{y}$ & & $\mathrm{y}$ \\
\hline LEP_2H_CS_ratios.dat & $\mathrm{y}$ & $\mathrm{y}$ & & \\
\hline TEV_H_Ojet_partCS_ratios.dat & $\mathrm{y}$ & & $\mathrm{y}$ & $\mathrm{y}$ \\
\hline TEV_H_1jet_partCS_ratios.dat & $\mathrm{y}$ & & $\mathrm{y}$ & $\mathrm{y}$ \\
\hline TEV_HW_partCS_ratios.dat & $\mathrm{y}$ & & $\mathrm{y}$ & $\mathrm{y}$ \\
\hline TEV_HZ_partCS_ratios.dat & $\mathrm{y}$ & & $\mathrm{y}$ & $\mathrm{y}$ \\
\hline TEV_H_vbf_hadCS_ratios.dat & $\mathrm{y}$ & & $\mathrm{y}$ & $\mathrm{y}$ \\
\hline TEV_1H_hadCS_ratios.dat & $\mathrm{y}$ & & $\mathrm{y}$ & $\mathrm{y}$ \\
\hline BR_1H.dat & $\mathrm{y}$ & $\mathrm{y}$ & $\mathrm{y}$ & $\mathrm{y}$ \\
\hline BR_2H.dat & $\mathrm{y}$ & $\mathrm{y}$ & & \\
\hline additional.dat (optional) & $\mathrm{y}$ & $\mathrm{y}$ & $\mathrm{y}$ & $\mathrm{y}$ \\
\hline
\end{tabular}

Table 15

List of input files, specifying which values of whichexpt each input file is relevant to (marked by ' $y$ ').

\section{Examples}

The HiggsBounds package includes a full set of sample input files for the case $n_{H}=3$, contained in the folder example. Each filename is prefixed with nH3.. To run the command-line version of HiggsBounds with these files as input, use, for example,

./configure

make

./HiggsBounds LandT effC 3 'example/nH3_'

where the values of whichexpt and whichinput can be varied as desired.

\subsection{Website version}

The website allows the user to select the required number of neutral Higgs bosons and then generates a html form accordingly. The values of whichinput 


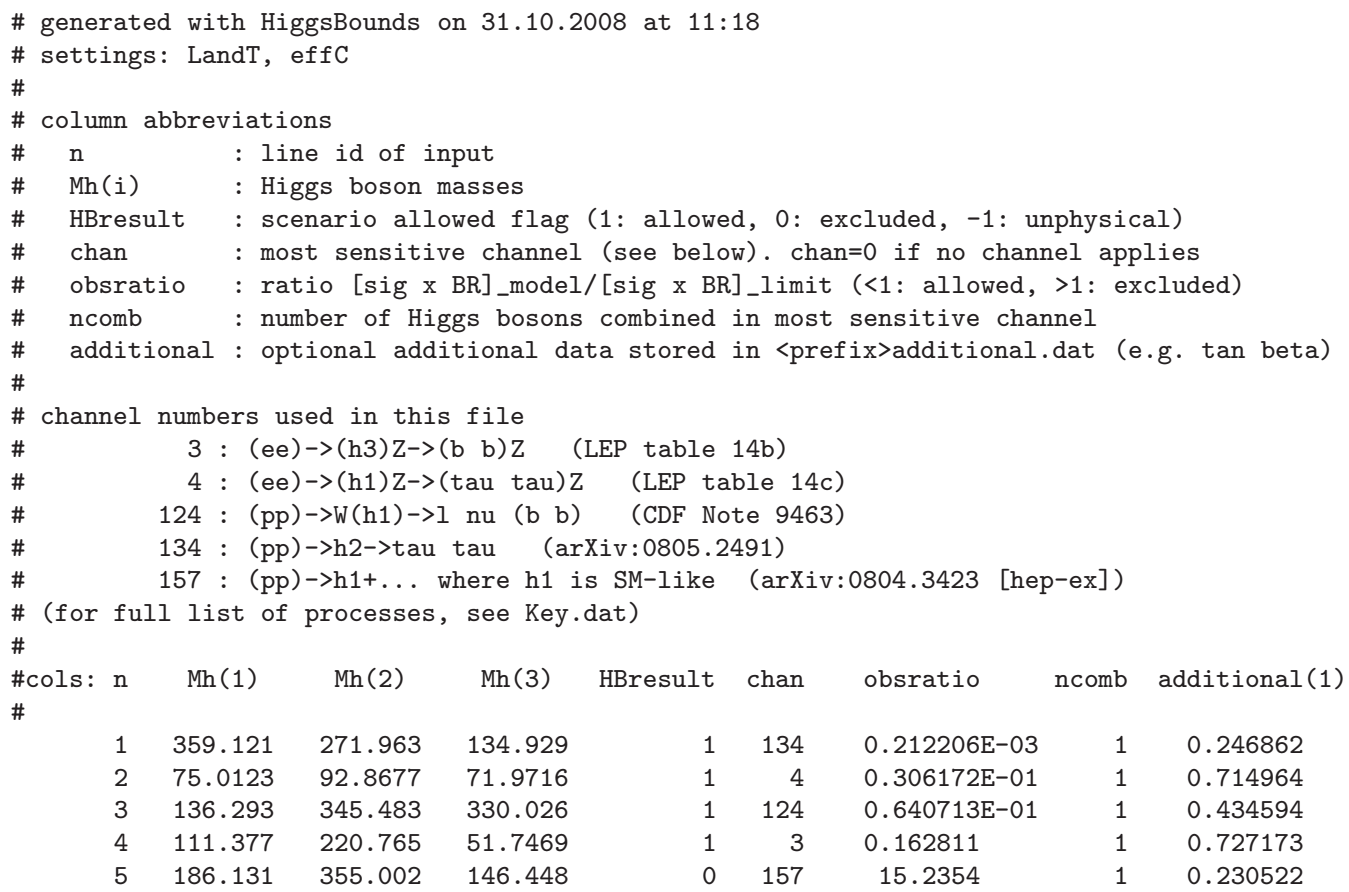

Fig. 2. Sample output file (written to <prefix $>$ HiggsBounds_Results . dat)

and whichexpt can be chosen and the appropriate theoretical input entered. HiggsBounds will then be called with these settings and the result outputted to screen. The website version contains the additional feature that it notifies the user about the processes with the second and third highest statistical sensitivities and the values of obsratio for these processes. This is designed to give guidance to the user who, for example, wishes to find an excluded region iteratively by adjusting the input quantities.

The website also contains a selection of pre-filled html forms as examples, including entries for the Standard Model, the fermiophobic Higgs model and the MSSM with real and complex parameters.

\section{Examples of use}

\subsection{Re-evaluation of SM exclusion limit with an improved SM prediction}

Recently, an improved prediction for the Higgs-boson production via gluon fusion appeared [58, which incorporates previously unknown mixed QCDelectroweak radiative corrections to this process as well as other known relevant corrections [59]. It turns out that those corrections are relevant, increasing the SM cross section prediction by $7-10 \%$ compared to the cross section prediction currently used by the Tevatron collaborations, when the MRST 2006 

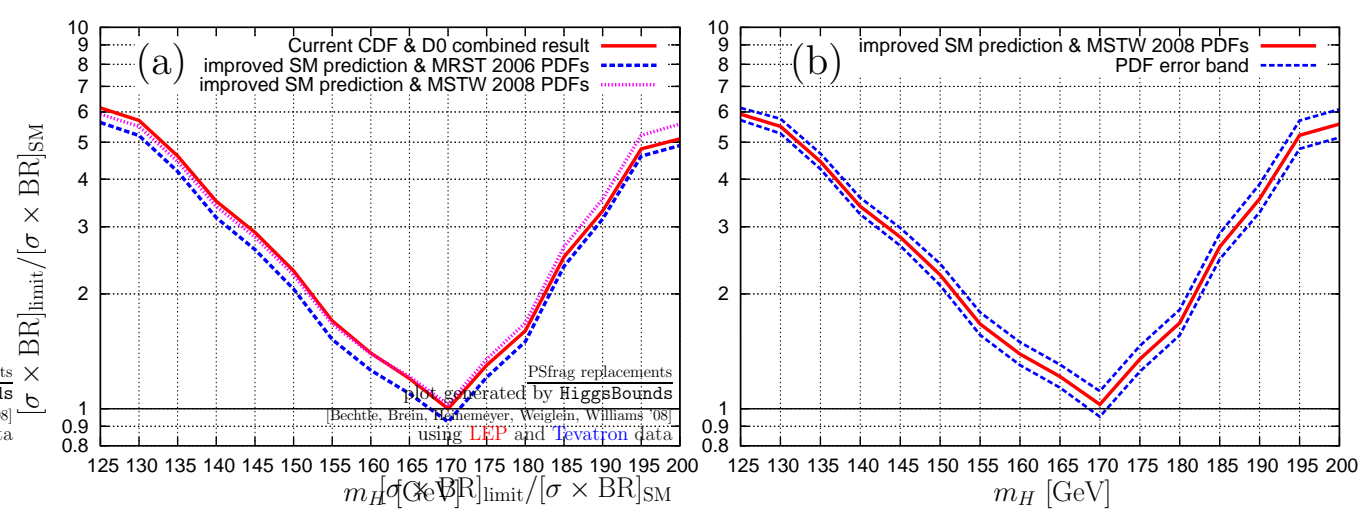

Fig. 3. Ratio $Q_{o b s} / Q_{S M}$ for the most sensitive channel as a function of the Higgs mass $m_{H}$ : (a) The current best available SM combined analyses of CDF and DØ (solid line) is compared to a re-evaluation using the recently improved SM prediction for the gluon fusion process with MRST 2006 PDFs (dashed line) and MSTW 2008 PDFs (dotted line) [58]; (b) The impact of the PDF uncertainty on the exclusion limit using the improved SM prediction [58] is shown.

NNLO parton distribution functions (PDFs) [60] are used. However, very recently, an updated set of PDFs, MSTW 2008 [54], appeared which show a decrease in the low- $x$ gluon distribution. In an updated version of Ref. 58 the combined effect of using the improved prediction for the SM cross section and the latest set of MSTW 2008 NNLO PDFs has been found to result in a downward shift of the SM cross section prediction in the region where the Tevatron searches have the highest sensitivity by $4-6 \%$ with respect to the cross section prediction used by the Tevatron collaborations.

With HiggsBounds the impact of such changes in the SM cross section prediction on the SM exclusion limit can easily be assessed. Fig. 3 shows the result of a re-evaluation of the Tevatron exclusion limits. In Fig. 3(a), the limit obtained using the cross section predictions of [58] with MRST 2006 NNLO PDFs and MSTW 2008 NNLO PDFs are compared with the published limits from the CDF and DØ SM combined analyses (48] for $m_{H}<155 \mathrm{GeV}$ and [49] for $m_{H}>155 \mathrm{GeV}$ ) which do not include this improved prediction. While in the published Tevatron result the cross section limit just touches the SM prediction for $m_{H} \approx 170 \mathrm{GeV}$, the updated SM cross section prediction exceeds the cross section limit within an interval of about $5 \mathrm{GeV}$ if MRST 2006 PDFs are used. However, when the most up-to-date MSTW 2008 PDFs are used, the cross section prediction is lower than the one obtained by the Tevatron collaborations for $m_{H}$ above about $160 \mathrm{GeV}$, resulting in a reduction of the exclusion power. With the updated prediction, no value of the SM Higgs boson mass is excluded at the 95\% C.L. Fig. 3(b) shows how the comparison between the SM cross section prediction and the exclusion limits from the Tevatron is affected by PDF uncertainties. The solid line shows the result based on the updated SM cross section prediction using MSTW 2008 PDFs, while the dashed lines indicate the PDF uncertainties as given in Ref. [58]. 
It can be clearly seen that currently no firm exclusion of any SM Higgs mass values at the $95 \%$ C.L. can be established from the exclusion bounds obtained at the Tevatron once PDF uncertainties on the SM cross section prediction are taken into account.

\subsection{Fourth Generation Model}

A very simple example of physics beyond the SM is a model which extends the SM by a fourth generation of heavy fermions [61]. In particular, the masses of the 4th generation quarks and leptons are assumed to be (much) heavier than the mass of the top-quark. In this case, the effective coupling of the Higgs boson to two gluons is three times larger than in the SM. No other coupling, relevant to LEP and Tevatron searches, changes significantly 8 . Essentially, only the partial decay width $\Gamma(H \rightarrow g g)$ changes by a factor of 9 and, with it, the total Higgs width and therefore all the decay branching ratios [62].

We can easily calculate the new total decay width and the relevant decay branching ratios using the above information and the built-in functions to calculate SM quantities using the relations:

$$
\begin{aligned}
\Gamma_{\mathrm{SM}}(H \rightarrow g g) & =\mathrm{BR}_{\mathrm{SM}}(H \rightarrow g g) \Gamma_{\mathrm{tot}}^{\mathrm{SM}}(H), \\
\Gamma_{\text {model }}(H \rightarrow g g) & =9 \Gamma_{\mathrm{SM}}(H \rightarrow g g), \\
\Gamma_{\text {tot }}^{\text {model }}(H) & =\Gamma_{\text {tot }}^{\mathrm{SM}}(H)-\Gamma_{\mathrm{SM}}(H \rightarrow g g)+\Gamma_{\text {model }}(H \rightarrow g g) .
\end{aligned}
$$

It is then very simple to test the model with HiggsBounds using the effective coupling input.

In Fig. 4 we show a Fortran 77 code example which uses HiggsBounds for the calculation of the Higgs mass dependence of the ratio $Q_{\text {model }} / Q_{\text {obs }}$ for the SM and our simple Fourth Generation Model scenario. From the results returned by the subroutine run_HiggsBounds_effC, the set (mh, HBresult, chan, obsratio) is stored in a file, separately for the two models.

The results obtained by this sample program are presented in Fig. 5, which shows the ratio $\left(Q_{\text {model }} / Q_{\text {obs }}\right)^{-1}=[\sigma \times \mathrm{BR}]_{\text {obs. limit }} /[\sigma \times \mathrm{BR}]_{\text {model }}$ as a function of the Higgs mass $m_{H}$ for the SM (solid lines) and the Fourth Generation Model (dashed lines). For the SM cross section we use here the prediction employed by the Tevatron collaborations, see the discussion above concerning

8 The effective Higgs-photon-photon coupling is suppressed by a factor of about $\frac{1}{9} \Gamma_{\text {tot }}^{\text {model }}(H) / \Gamma_{\text {tot }}^{\mathrm{SM}}(H)$ for a light Higgs $H$ [62]. The exact suppression factor is irrelevant here, as the Higgs decay into $\gamma \gamma$ can only lead to relevant search topologies at the Tevatron if it is enhanced by more than an order of magnitude compared to the SM. 


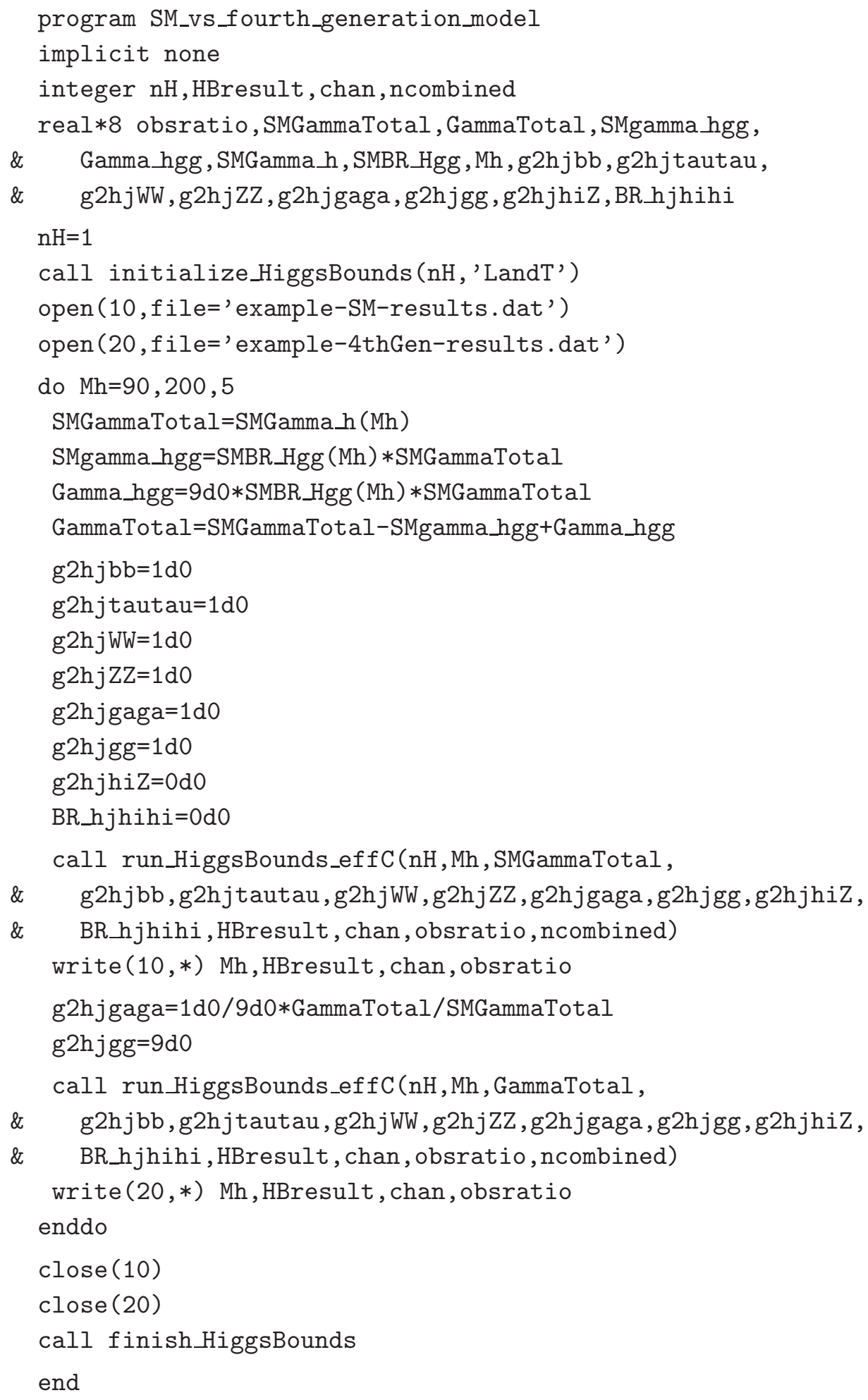

Fig. 4. Fortran77 code example-SM_vs_4thGen.F which calculates the ratio $Q_{\text {model }} / Q_{\text {obs }}$ (obsratio) for the most sensitive Higgs search topology for the $S M$ and a simple Fourth Generation Model as a function of the Higgs mass (Mh). This program has been used to produce the plot shown in Fig. 5. 


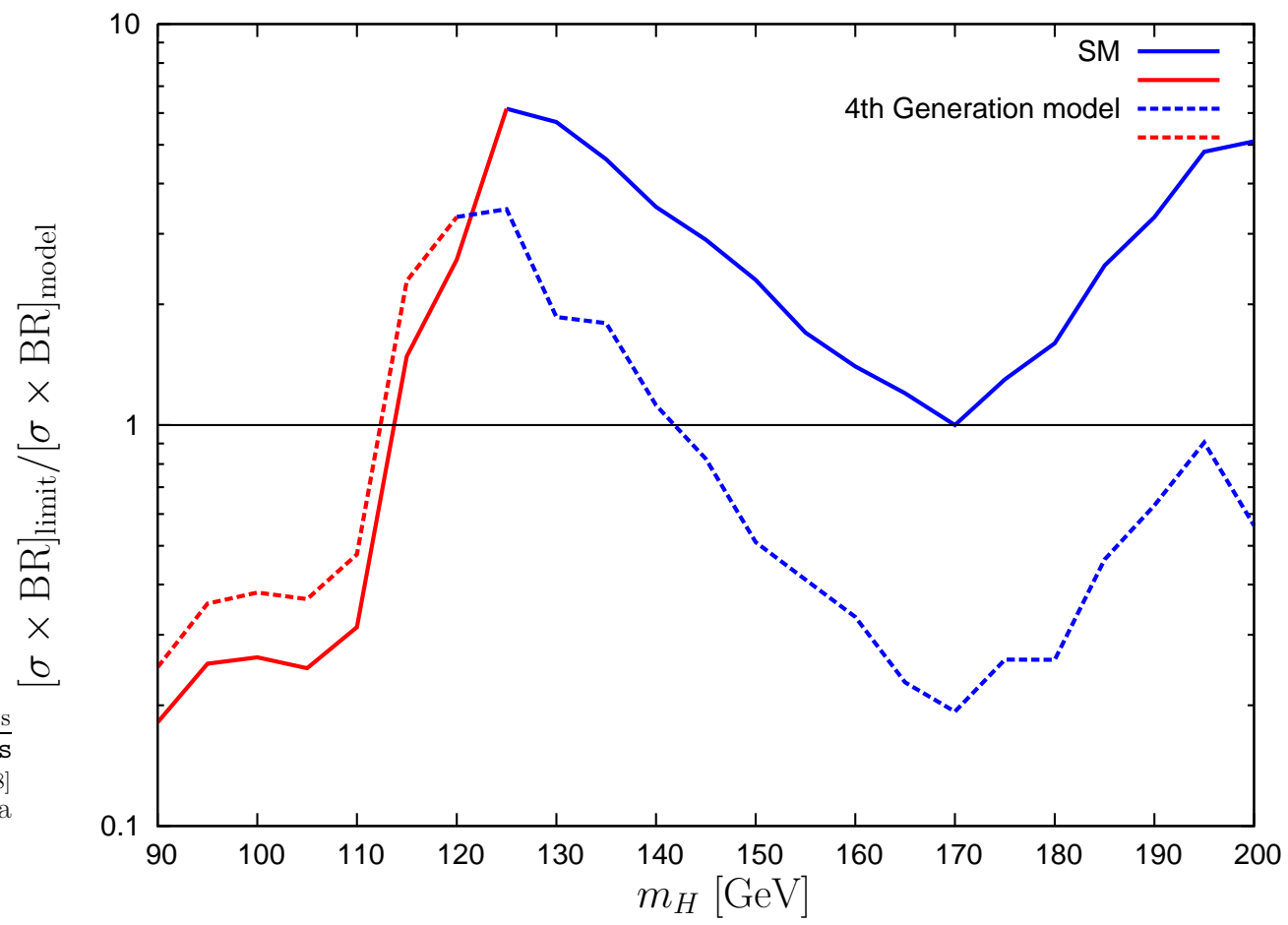

Fig. 5. Ratio $Q_{\text {obs }} / Q_{\text {model }}$ for the most sensitive channel as a function of the Higgs mass $m_{H}$ : 4th Generation Model versus SM. The colours indicate whether the most sensitive search channel is from LEP (lighter grey) or the Tevatron (darker grey).

an update of this prediction. Exclusion at 95\% C.L. is given if the value of $\left(Q_{\text {model }} / Q_{\text {obs }}\right)^{-1}$ drops below 1 . Both curves have sections in red colour (lighter grey), where the LEP results provide the most sensitive search topology, and in blue colour (darker grey), where the most sensitive search topology is provided by the Tevatron results. As expected, the LEP results have the highest sensitivity in the low-mass region up to the kinematical limit of about $115 \mathrm{GeV}$, while for higher mass values the Tevatron results take over.

The SM results show the familiar exclusion of Higgs masses below $\approx 115 \mathrm{GeV}$ from LEP and the small excluded region at about $m_{H}=170 \mathrm{GeV}$ from the latest Tevatron SM combined analysis [49] using the results of the slightly more conservative results obtained by the Bayesian method (see the discussion above for an update of the cross-section prediction employed here). For the Fourth Generation Model the LEP exclusion is a bit weaker than for the SM, because at low Higgs mass the larger branching ratio for Higgs decays into gluon pairs diminishes the $b \bar{b}$ and $\tau^{+} \tau^{-}$branching ratios. In the region above $115 \mathrm{GeV}$, the most sensitive search topology for the Fourth Generation Model Higgs is $p \bar{p} \rightarrow H \rightarrow W^{+} W^{-} \rightarrow l^{+} l^{\prime-}$ throughout the displayed range. Because of the enhancement of the signal cross section due to the enhanced coupling of the Higgs boson to gluons, the region excluded by the Tevatron results extends from about $145 \mathrm{GeV}$ to the end of the displayed range. Because of the SMlikeness criterion, the single topology analyses [3940] apply, instead of the 
SM combined analysis, for the Fourth Generation Model. Disregarding this criterion would change the $\left(Q_{\text {model }} / Q_{\text {obs }}\right)^{-1}$ values in the Tevatron exclusion region to almost exactly $1 / 9$ times the $\mathrm{SM}$ values.

\section{$4.3 M S S M$}

\section{Theoretical background}

The MSSM Higgs sector consists of two scalar weak isospin doublets and possesses therefore five physical Higgs bosons. At tree-level these are the $\mathcal{C} \mathcal{P}$ even $h$ and $H$ (with $m_{h}<m_{H}$ ), the $\mathcal{C P}$-odd $A$ and the charged $H^{ \pm}$. The Higgs sector of the MSSM can be expressed at lowest order in terms of $M_{Z}$, $M_{A}$ (or $M_{H^{ \pm}}$) and $\tan \beta \equiv v_{2} / v_{1}$, the ratio of the vacuum expectation values of the two doublets. All other masses and mixing angles can therefore be predicted. The tree-level masses and couplings are strongly affected by higherorder corrections 63. In the real MSSM (rMSSM), i.e. the model without $\mathcal{C P}$-violation, the higher-order corrected masses are denoted as $M_{h}, M_{H}$ and $M_{A}$. In the MSSM with complex phases (cMSSM) the three neutral Higgs bosons can mix and the masses are denoted as $M_{h_{1}}, M_{h_{2}}, M_{h_{3}}$ (with $M_{h_{1}}<$ $\left.M_{h_{2}}<M_{h_{3}}\right)$.

In order to ensure that the external Higgs bosons have the correct on-shell properties, the tree-level Higgs states have to be supplemented with Higgspropagator corrections (which are often the dominant corrections). This is done with the help of the $\mathbf{Z}$ matrix [17,64], a non-unitary matrix, which mixes between the Higgs tree-level mass eigenstates (in the order $h, H, A$ ) and the loop-corrected mass eigenstates

$$
\left(\begin{array}{l}
h_{1} \\
h_{2} \\
h_{3}
\end{array}\right)=\mathbf{Z} \cdot\left(\begin{array}{l}
h \\
H \\
A
\end{array}\right) .
$$

In the rMSSM, setting $h_{1}=h, h_{2}=H, h_{3}=A$, one has

$$
Z_{31}=Z_{32}=Z_{13}=Z_{23}=0, Z_{33}=1
$$

In the following we give the formulas valid for the more general cMSSM, where the special case of the rMSSM can be derived with the help of Eq. (60). The normalised cross sections $\sigma_{h_{i} Z}^{\text {norm }}, \sigma_{h_{j} h_{i}}^{\text {norm }}$, defined through Eqs. (11), (18) and (20) as part of the input needed by HiggsBounds, can then be found using the elements of the matrix $\mathbf{Z}$ 


$$
\begin{aligned}
\sigma_{h_{i} Z}^{\text {norm }}= & \left|Z_{i 1} \sin (\beta-\alpha)+Z_{i 2} \cos (\beta-\alpha)\right|^{2} \\
\sigma_{h_{j} h_{i}}^{\text {norm }}= & \mid Z_{i 3}\left(Z_{j 1} \cos (\beta-\alpha)-Z_{j 2} \sin (\beta-\alpha)\right) \\
& -\left.Z_{j 3}\left(Z_{i 1} \cos (\beta-\alpha)-Z_{i 2} \sin (\beta-\alpha)\right)\right|^{2},
\end{aligned}
$$

where $\alpha$ is the angle that diagonalises the $\mathcal{C P}$-even Higgs sector at tree-level. It should be noted that for two identical Higgs bosons this leads to $\sigma_{h_{i} h_{i}}^{\text {norm }}=0$ and that the following approximate relations hold:

$$
\begin{aligned}
\sigma_{h_{1} Z}^{\text {norm }}+\sigma_{h_{2} Z}^{\text {norm }}+\sigma_{h_{3} Z}^{\text {norm }} & \simeq 1, \\
\sigma_{h_{k} Z}^{\text {norm }} & \simeq \sigma_{h_{j} h_{i}}^{\text {norm }},
\end{aligned}
$$

where $i, j, k$ are all different 9 .

\section{Limits for the real MSSM}

In Ref. [12] the final results for the LEP Higgs searches in the MSSM were published. In the case of the rMSSM they had been obtained in four benchmark scenarios: the $m_{h}^{\max }$, no-mixing, small $\alpha_{\text {eff }}$ and gluophobic Higgs scenarios (including also some variations), see Ref. 65] for a motivation and detailed definition of these scenarios. The computer code used at that time was FeynHiggs 2.0, and in most cases the top-quark mass was fixed to $m_{t}=$ $174.3 \mathrm{GeV}$. The limits had been obtained by a combination of all available channels.

Here we present the limits obtained by HiggsBounds using the latest version (2.6.4) of FeynHiggs to calculate the required model input and choosing different values for $m_{t}$. Note that the limits given here are in the narrow-width approximation, as discussed in Section 2.1.

In the left column of Fig. 6 we show the LEP and Tevatron exclusion bounds as obtained with HiggsBounds in the $m_{h}^{\max }(\mathrm{a})$, no-mixing (b), small $\alpha_{\text {eff }}$ (c) and gluophobic Higgs scenario (d) in the $M_{A}$-tan $\beta$ plane for the current topquark mass value, $m_{t}=172.4 \mathrm{GeV}$ [66]. In the right column of Fig. 6] we show the most sensitive channels for each point in the $M_{A}-\tan \beta$ plane for the same four scenarios (e)-(h) (the colour code is given in the caption).

The four scenarios show similar features in the excluded regions, as presented in the left-hand column. Very low $M_{A}$ values are excluded by LEP searches for all $\tan \beta$ values. This is due to the channel $e^{+} e^{-} \rightarrow h A \rightarrow b \bar{b} b \bar{b}$ as can be seen in the right column of Fig. 6. In this region the $h Z Z$ coupling is suppressed. Only at very low $\tan \beta$ values the channel $e^{+} e^{-} \rightarrow h Z, h \rightarrow b \bar{b}$ has

9 Note that these relations become equalities if a unitary Higgs mixing matrix is used. See, e.g., Ref. [17]. 

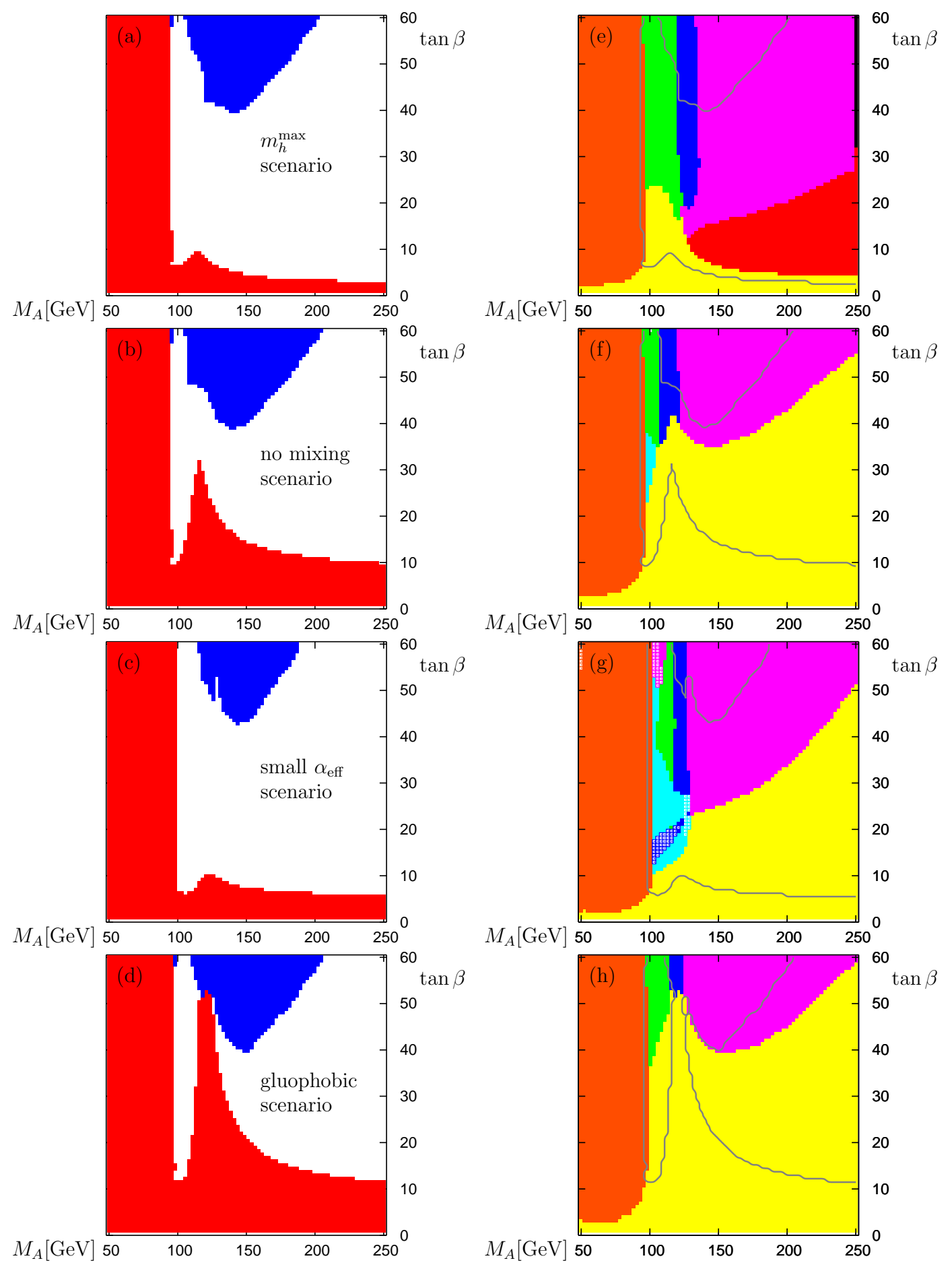

Fig. 6. Left column [(a),(b),(c),(d)]: 95\% C.L. level excluded regions in the $M_{A}$-tan $\beta$ plane for the the four rMSSM benchmark scenarios. The colours indicate exclu-

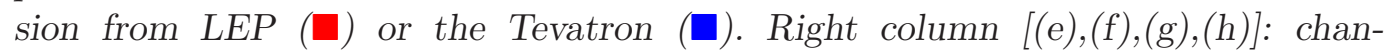
nel of highest statistical sensitivity: yellow $(\square): e^{+} e^{-} \rightarrow h Z, h \rightarrow b \bar{b}$; cyan $(\square)$ : $e^{+} e^{-} \rightarrow H Z, H \rightarrow b \bar{b}$; cyan $(\cdot): e^{+} e^{-} \rightarrow h Z / H Z, h / H \rightarrow b \bar{b}$; orange $(\square / \bullet):$

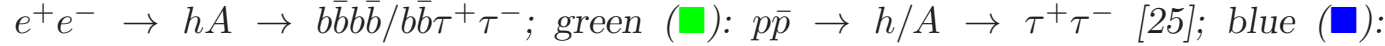

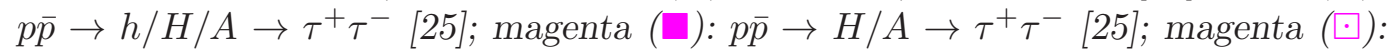

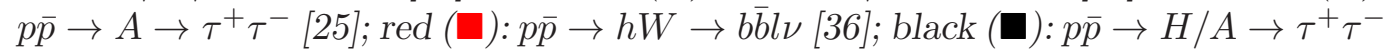
[24]; blue (घ): $p \bar{p} \rightarrow H \rightarrow W^{+} W^{-}$[39]. The contour borders the excluded regions. 
the highest statistical sensitivity, but still with a somewhat suppressed $h Z Z$ coupling. At around $M_{A} \approx 100 \mathrm{GeV}$ the kinematical limit for the $h A$ channel is reached and other channels take over. There the exclusion drops to a relatively low excluded $\tan \beta$ value, slightly different in the four scenarios. At around $M_{A} \approx 115 \mathrm{GeV}$ the exclusion in the $e^{+} e^{-} \rightarrow h Z, h \rightarrow b \bar{b}$ channel becomes SMlike, and Higgs bosons up to the kinematical limit can be excluded by LEP searches, reaching $\tan \beta=8,30,10,50$ in the $m_{h}^{\max }$, no-mixing, small $\alpha_{\text {eff }}$ and gluophobic Higgs scenario, respectively. In the latter one also larger values could be excluded, but the Tevatron channels become more important, see below. For larger $M_{A}$ values the LEP exclusion drops to a lower $\tan \beta$ value between 3 and 11, depending on the scenario. These $\tan \beta$ values correspond to $M_{h} \approx 208 \mathrm{GeV}-M_{Z}$, i.e. the kinematical limit and are excluded for all $M_{A}$ values above $\sim 115 \mathrm{GeV}$.

The Tevatron searches exclude a parameter space, depending on the scenario, between $M_{A}=110 \mathrm{GeV}$ and $170 \mathrm{GeV}$, and between $\tan \beta=39$ and 60 (where we stopped our scan, but in principle larger values can be excluded). The relevant channels are $p \bar{p} \rightarrow h / H / A+X, h / H / A \rightarrow \tau^{+} \tau^{-}$, see the right-hand column of Fig. 6. The variation in the colour here only indicates which Higgs bosons contribute together to the signal. At low $M_{A}$ (green) one has $M_{h} \approx M_{A}$, at intermediate $M_{A}$ (dark blue) we find $M_{h} \approx M_{A} \approx M_{H}$, and at higher $M_{A}$ values one generically has $M_{A} \approx M_{H}$. Consequently, two or three neutral Higgs bosons can contribute to the signal. Two other Tevatron channels have the largest exclusion potential in some part of the $M_{A}-\tan \beta$ parameter space. Within the $m_{h}^{\max }$ scenario $p \bar{p} \rightarrow h W \rightarrow b \bar{b} l \nu$ is the most sensitive channel for $M_{A} \gtrsim 130 \mathrm{GeV}$ and intermediate $\tan \beta$, see plot (e) in Fig. 6. In the small $\alpha_{\text {eff }}$ scenario $p \bar{p} \rightarrow H \rightarrow W^{+} W^{-}$with the $W$ s decaying leptonically is the most sensitive channel for low $M_{A}$ and intermediate $\tan \beta$, see plot (g) of Fig. 6.

As mentioned before, the original exclusion bounds in Ref. 12 had been obtained for $m_{t}=174.3 \mathrm{GeV}$. The most prominent effect of increasing the topquark mass from $m_{t}=172.4 \mathrm{GeV}$ to $m_{t}=174.3 \mathrm{GeV}$ is an increase in $M_{h}$ with $\Delta M_{h} / \Delta m_{t} \lesssim 1$ [67]. This reduces the reach in the $M_{A}-\tan \beta$ plane at LEP for the $e^{+} e^{-} \rightarrow h Z$ channels. This effect can be observed to some extent in all four scenarios. In Fig. 7 we show the exclusion bounds as obtained with HiggsBounds with $m_{t}=174.3 \mathrm{GeV}$ in the $m_{h}^{\max }$ scenario in the $M_{h}-\tan \beta$ plane. This plot can be compared with Fig. 9(b) in the original LEP publication [12, which, however, terminated at $\tan \beta=40$, whereas the plot shown here extends up to $\tan \beta=60$. The values of $M_{h}$ reached in Fig. 7 are smaller by $\sim 2 \mathrm{GeV}$ as compared to the original LEP figure, which is due to the newer version of FeynHiggs employed here. Apart from this, the LEP exclusion areas are almost identical in the two figures. This shows how well in this case our procedure (based on the LEP data for single topological cross sections) reproduces the full LEP analysis where different search channels were combined. In addition, we also find a region excluded by the Tevatron Higgs searches at 


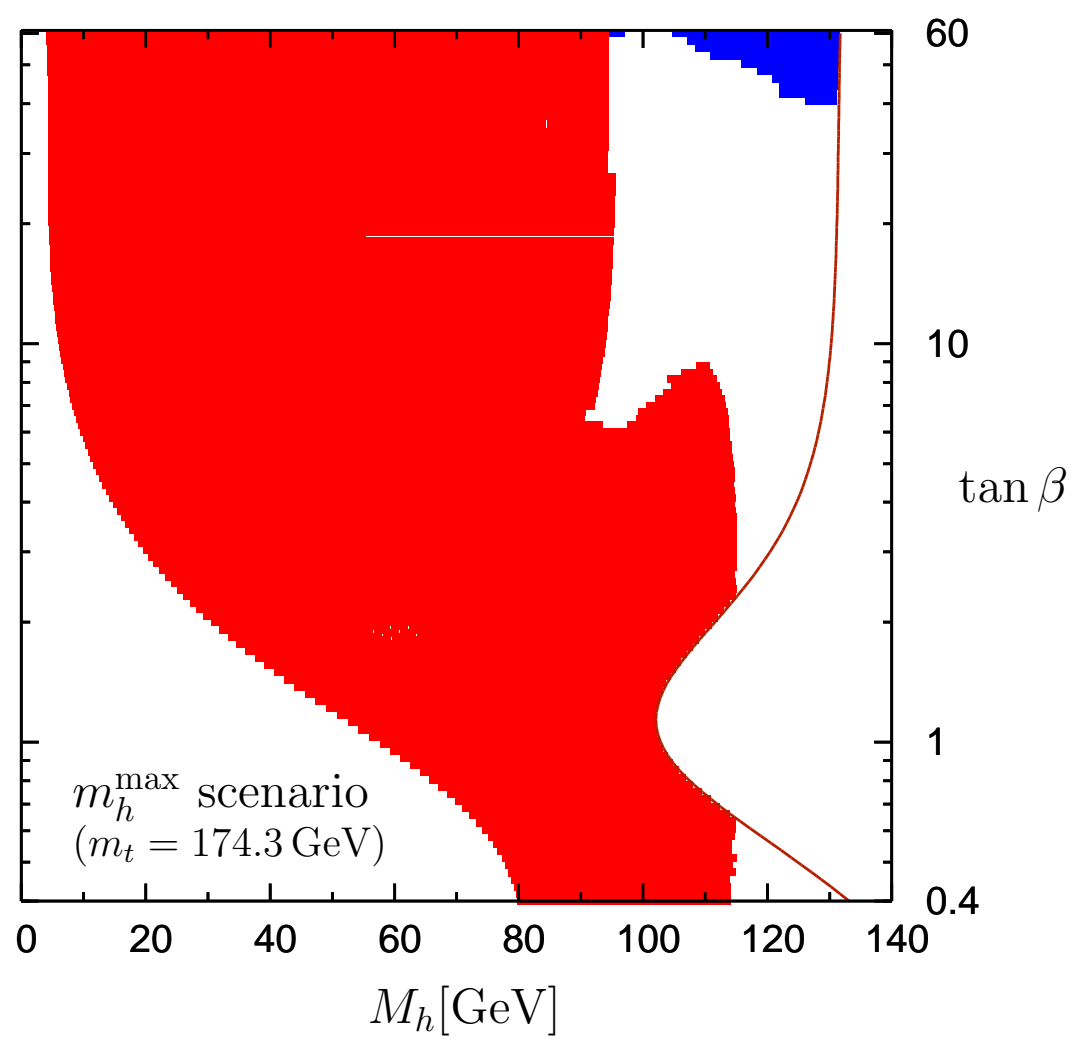

Fig. 7. Parameter region in the $M_{h}$-tan $\beta$ plane for the $m_{h}^{\max }$ scenario of the rMSSM which is excluded at the $95 \%$ C.L. The mass of the top quark has been set to 174.3 $\mathrm{GeV}$. The colours indicate whether the most sensitive search channel which leads to exclusion is from LEP (lighter grey) or the Tevatron (darker grey). The solid line shows the theoretical upper bound on $M_{h}$ in the $m_{h}^{\max }$ scenario.

$\tan \beta \gtrsim 40$ and $M_{h} \gtrsim 100 \mathrm{GeV}$. These results from the Tevatron were not yet available when Ref. [12] was published.

\section{LEP limits for the complex MSSM in the CPX scenario}

Ref. [12] also published the results of the LEP Higgs searches in the CPX scenario, which is a $\mathcal{C P}$-violating MSSM benchmark scenario [68]. This analysis incorporated Higgs sector predictions from the programs $\mathrm{CPH}$ [68,69] and FeynHiggs 2.0 [14,15,16, 17].

More recently, in Ref. [70], a preliminary version of HiggsBounds was used in conjunction with full 1-loop vertex corrections for the $h_{j} \rightarrow h_{i} h_{i}$ and $h_{j} \rightarrow f \bar{f}$ decays to examine the effect of these corrections on the LEP exclusions in the CPX scenario. In Ref. [70], Higgs masses and the Higgs propagator corrections (as contained in the $\mathbf{Z}$ matrix) were calculated using renormalised Higgs selfenergies from FeynHiggs 2.6.4. 


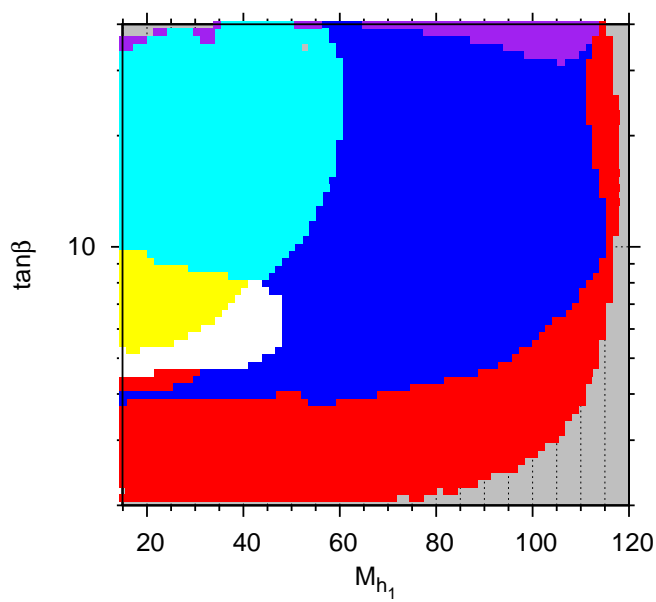

(a)

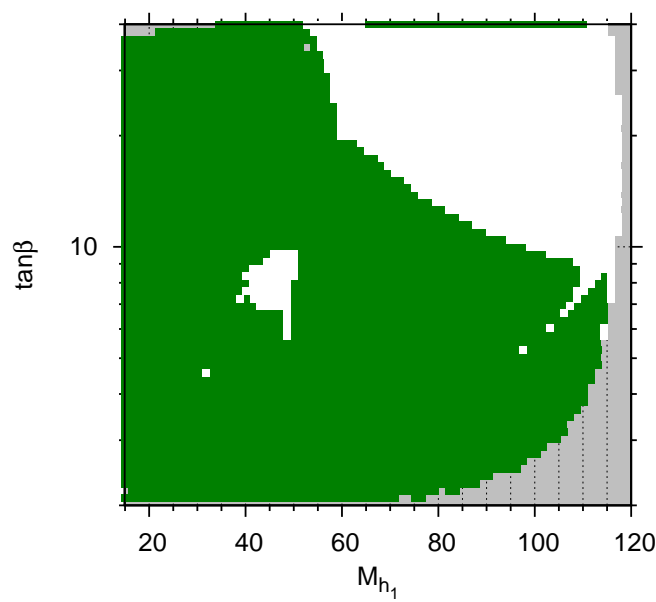

(b)

Fig. 8. Coverage of the LEP Higgs searches in the $M_{H_{1}}-\tan \beta$ plane of the CPX scenario. Plot (a) shows the channels that are predicted to have the highest statistical sensitivity for setting an exclusion limit. The colour codings are: red $(\mathbf{\square})=\left(h_{1} Z\right) \rightarrow(b \bar{b} Z)$, blue $(\mathbf{\square})=\left(h_{2} Z\right) \rightarrow(b \bar{b} Z)$, white $(\square)=$ $\left(h_{2} Z\right) \rightarrow\left(h_{1} h_{1} Z\right) \rightarrow(b \bar{b} b \bar{b} Z)$, cyan $(\square)=\left(h_{2} h_{1}\right) \rightarrow(b \bar{b} b \bar{b})$, yellow $(\square)=$ $\left(h_{2} h_{1}\right) \rightarrow\left(h_{1} h_{1} h_{1}\right) \rightarrow(b \bar{b} b \bar{b} b \bar{b})$, purple $(\mathbf{\square})=$ other channels. Plot (b) shows the parameter regions excluded at the $95 \%$ C.L. by the topological cross section limits obtained at LEP [1112]. The colour codings are: green $(\square)=$ excluded, white $(\square)=$ unexcluded. In both plots, the grey $(\square)$ peripheral areas are theoretically inaccessible using the input parameters associated with the CPX scenario.

The results shown in Fig. 8 have been calculated using the convention 10 and method described in Ref. [70]. However, the results shown here include two significant updates: we use $m_{t}=172.6 \mathrm{GeV}$ rather than $m_{t}=170.9 \mathrm{GeV}$ and we use a newer version of FeynHiggs to calculate the renormalised Higgs self-energies, which contains the correct combination of the $\tan \beta$-enhanced contributions to the Higgs-boson self-energies in the complex MSSM with the one-loop corrections for all options concerning the two-loop contributions.

In Fig. 8 (a), we show the channels with the highest statistical sensitivity for each point in CPX parameter space. The process $\left(h_{1} Z\right) \rightarrow(b \bar{b} Z)($ red $) 11$ dominates in regions where the $h_{1} Z Z$ effective coupling is significant. Adjacent to this region is an area of parameter space with a relatively large $h_{2} Z Z$ effective coupling and therefore the processes $\left(h_{2} Z\right) \rightarrow(b \bar{b} Z)$ (blue) and $\left(h_{2} Z\right) \rightarrow$ $\left(h_{1} h_{1} Z\right) \rightarrow(b \bar{b} b \bar{b} Z)$ (white) have the highest statistical sensitivity in this region. The processes $\left(h_{2} h_{1}\right) \rightarrow(b \bar{b} b \bar{b})($ cyan $)$ and $\left(h_{2} h_{1}\right) \rightarrow\left(h_{1} h_{1} h_{1}\right) \rightarrow(b \bar{b} b \bar{b} b \bar{b})$

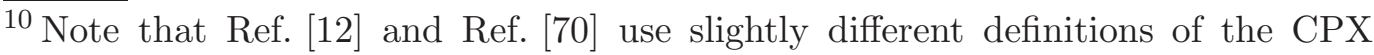
scenario (see Ref. [70] for a full description of these differences).

${ }^{11}$ All processes discussed in this subsection are LEP processes. Therefore, for brevity, we do not include the initial state explicitly in the process descriptions.
} 
(yellow) dominate in the region where the $h_{3} Z Z$ effective coupling is relatively large (and therefore also the $h_{1} h_{2} Z$ effective coupling, as predicted by Eq. (64)).

In the region of Fig. 8 (a) where $M_{h_{1}} \lesssim 45 \mathrm{GeV}, \tan \beta \gtrsim 4$, the total decay width of $h_{2}$ is dominated by the partial decay width $h_{2} \rightarrow h_{1} h_{1}$. This has a very significant effect on the exclusion prospects in this region. As Fig. 8 (a) shows, this region is partly covered by the $\left(h_{2} Z\right) \rightarrow\left(h_{1} h_{1} Z\right) \rightarrow(b \bar{b} b \bar{b} Z)$ (white) and $\left(h_{2} h_{1}\right) \rightarrow\left(h_{1} h_{1} h_{1}\right) \rightarrow(b \bar{b} b \bar{b} b \bar{b})$ (yellow) processes, which directly involve the $h_{2} \rightarrow h_{1} h_{1}$ decay. However, searching for these topologies is very challenging experimentally as, for example, the $\left(h_{2} h_{1}\right) \rightarrow\left(h_{1} h_{1} h_{1}\right) \rightarrow(b \bar{b} b \bar{b} b \bar{b})$ process gives rise to complex final states involving six jets. Additionally, processes directly involving the $h_{2} \rightarrow b \bar{b}$ decay (such as $\left(h_{2} Z\right) \rightarrow(b \bar{b} Z)$ (blue)) are more difficult to detect in this region because of the suppression of the $h_{2} \rightarrow b \bar{b}$ branching ratio due to the large $h_{2} \rightarrow h_{1} h_{1}$ partial decay width.

Fig. 8 (b) shows the areas of CPX parameter space which have been excluded by the LEP Higgs searches at the $95 \%$ C.L. (green). There are two sizable unexcluded region: 12 (white), at $M_{h_{1}} \gtrsim 50 \mathrm{GeV}$, $\tan \beta \gtrsim 8$ and a "hole" at $M_{h_{1}} \approx 45 \mathrm{GeV}, \tan \beta \approx 8$. Both of these regions occur in parts of parameter space where processes involving the topology $\left(h_{i} Z\right) \rightarrow(b \bar{b} Z)$ predominantly have the highest statistical sensitivity, and the second of these regions occurs in the part of the CPX parameter space which, as discussed above, was expected to prove challenging experimentally due to a suppressed $h_{2} \rightarrow b \bar{b}$ branching ratio. In addition, the two unexcluded regions are very sensitive to the slight excess in the LEP data for this topology at $89.6 \mathrm{GeV} \lesssim M_{h_{i}} \lesssim 107 \mathrm{GeV}$, where the observed limit is more than 1 sigma above the predicted limit based on simulations with no signal, see Ref. [12].

Comparison of Fig. 8 (a) with Fig. 8 (b) also shows that there are some unexcluded parameter points along boundaries between areas where different processes have the highest statistical sensitivity, for example, at $M_{h_{1}} \approx 100 \mathrm{GeV}$, $\tan \beta \approx 5$. Since in our analysis only the single channel having the highest statistical sensitivity can be used to set the $95 \%$ C.L. limit, the statistical information from all other decay and production channels in this region does not contribute. In these narrow regions, an analysis which can utilise more than one experimentally measured cross section limit for each parameter point (such as that performed in Ref. [12]) will have a higher exclusion power, and thus possibly be able to reduce the size of the unexcluded region.

\footnotetext{
$\overline{12}$ Note that we do not consider unexcluded regions to be significant if they only occur in a very small Higgs mass range and are situated on the borders of regions where different channels have the highest statistical sensitivity. As discussed in Section 2.1, we expect HiggsBounds to be less sensitive at these borders than a dedicated experimental analysis.
} 
Using HiggsBounds, it has therefore been possible to investigate the effects of recent improvements in the theoretical Higgs sector predictions on the LEP exclusions in the CPX scenario. In particular, since it provides information about the process with the highest statistical sensitivity, HiggsBounds should be helpful for the user to obtain some guidance on which effects are important for the exclusion or non-exclusion of certain parts of parameter space. HiggsBounds also provides a convenient way to investigate the dependence of the calculation on the model input parameters, such as the effect of varying the top-quark mass.

\section{Summary}

We have presented the code HiggsBounds, which is a tool to test whether the predictions arising from the Higgs sector of arbitrary types of theoretical models are in accordance with the existing exclusion bounds from the Higgs searches at LEP and the Tevatron. To this end, the experimental bounds on topological cross sections made available by the LEP and Tevatron collaborations have been incorporated as look-up tables into the program. In order to utilise this information to set exclusion bounds on the parameter space of a certain model one first needs to determine the search channel with the highest statistical sensitivity for setting an exclusion limit. This is done by comparing the expected limits (which have also been provided by the experimental collaborations), i.e. the exclusion bounds that one would obtain in the hypothetical case of an observed distribution with a pure background shape, with the model predictions. In order to ensure the correct statistical interpretation of the obtained exclusion bound as a 95\% C.L., the analysis has to be restricted to the single channel that possesses the highest statistical sensitivity for setting an exclusion limit. For this channel the program determines whether or not a certain parameter point is excluded at the 95\% C.L.

The experimental information incorporated into HiggsBounds consists primarily of limits on single topological cross sections, either from a single collaboration or combinations of the results from different collaborations. Some experimental combinations have been done by the collaborations only for the special case of a SM-type Higgs boson. These have the advantage of an enhanced statistical power. In order to determine whether experimental results on the searches for the SM Higgs can be used for constraining a certain parameter region of a new physics scenario, the "SM likeness" of the Higgs boson(s) of the model under study is evaluated. Most experimental results that are currently available have been obtained under the assumption that a narrow width approximation is valid for the Higgs boson under study. If this assumption is not valid the experimental exclusion bounds are modified. The current version of HiggsBounds is based on exclusion limits obtained in the narrow- 
width approximation. The proper treatment of experimental results, taking into account a non-negligible Higgs-boson width, will be incorporated in a forthcoming version of HiggsBounds. The exclusion bounds included in the HiggsBounds package will be updated as new results from the Higgs searches become available.

The predictions of the desired model, needed as input for HiggsBounds, have to be provided by the user. This can be done at various levels of sophistication, ranging from effective couplings to complete information on cross sections and branching ratios. Links to the widely used programs FeynHiggs and CPsuperH for Higgs-sector predictions in the MSSM are provided by default.

We have discussed various examples for running HiggsBounds and have given sample files for its input and output. In particular, we have considered the cases of the Standard Model, a model with a fourth generation of quarks and leptons and for the MSSM with and without $\mathcal{C P}$-violation.

The HiggsBounds code exists both in a Fortran 77 and Fortran 90 version. It can be operated in a command line mode, as a subroutine that can easily be linked to other codes, and as an online version, see

www.ippp.dur.ac.uk/HiggsBounds.

\section{Acknowledgements}

We thank the LEP collaborations ALEPH, DELPHI, L3 and OPAL and the Tevatron collaborations CDF and D $\varnothing$ for providing us with detailed information about their Higgs search results. In particular, we are grateful to Alexander Read and Peter Igo-Kemenes (LEP Higgs Working Group), Mark Owen (DØ), Tom Junk and Matthew Herndon (CDF) for very valuable discussions on their experiment's results. We thank Terrance Figy for providing us with SM predictions for the Higgs production cross section via VBF at the Tevatron separated in $W$ - and $Z$-fusion contribution. We would like to thank Philip Roffe and David Ambrose-Griffith for valuable computing support, and the IPPP (Durham, UK) for hosting the web version of HiggsBounds. Thanks also go to Lydia Heck, Sophy Palmer and Alison Fowler for their assistance in testing the code and to Valery Khoze for interesting discussions. KW was supported by the Helmholtz Alliance HA-101 'Physics at the Terascale'. This work has been supported in part by the European Community's Marie-Curie Research Training Network under contract MRTN-CT-2006-035505 'Tools and Precision Calculations for Physics Discoveries at Colliders' (HEPTOOLS) and MRTN-CT-2006-035657 'Understanding the Electroweak Symmetry Breaking and the Origin of Mass using the First Data of ATLAS' (ARTEMIS), by the Helmholtz Young Investigator Grant VH-NG-303, and by the DFG Collabo- 
rative Research Grant SFB 676 'Particles, Strings and the Early Universe'.

\section{A Determination of experimental $S_{95}$ values}

\section{A.1 The $C L_{s}$ method}

In order to calculate the experimental $S_{95}$ values, the modified frequentist method (or $C L_{s}$ method) is used. As a first step, using the experimental data binned in $i$ bins with $d_{i}$ observed data events, $s_{i}$ expected signal events for a given hypothesis of the signal, and $b_{i}$ background events, a test statistics

$$
Q=\prod_{i=1}^{j} \frac{e^{-\left(s_{i}+b_{i}\right)}\left(s_{i}+b_{i}\right)^{d_{i}}}{d_{i} !} / \frac{e^{-\left(b_{i}\right)}\left(b_{i}\right)^{d_{i}}}{d_{i} !}
$$

is defined, which orders the expected outcomes of test experiments according to their "signal-likeness". Then, confidence levels for the given hypothesis are calculated. The confidence level for the background hypothesis, $\mathrm{CL}_{b}$, is defined as the probability to obtain values of a test statistics $Q$ no larger than the observed value $Q_{\text {obs }}$, given a large number of hypothetical experiments with background processes only,

$$
\mathrm{CL}_{b}=P\left(Q \leq Q_{\text {obs }} \mid \text { background }\right) \text {. }
$$

Similarly, the confidence level for the signal+background hypothesis, $\mathrm{CL}_{s+b}$, is defined as the probability to obtain values of $Q$ smaller than observed, given a large number of hypothetical experiments with signal and background processes,

$$
\mathrm{CL}_{s+b}=P\left(Q \leq Q_{\text {obs }} \mid \text { signal }+ \text { background }\right) .
$$

In principle, $\mathrm{CL}_{s+b}$ can be used to exclude the signal+background hypothesis, given a model for Higgs boson production. However, this procedure may lead to the undesired possibility that a downward fluctuation of the background would allow a hypotheses to be excluded for which the experiment has no sensitivity due to the small expected signal rate. This problem is avoided by introducing the ratio

$$
\mathrm{CL}_{s}=\mathrm{CL}_{s+b} / \mathrm{CL}_{b} \text {. }
$$

Since $\mathrm{CL}_{b}$ is a positive number less than one, $\mathrm{CL}_{s}$ will always be greater than $\mathrm{CL}_{s+b}$ and the limit obtained in this way will therefore be conservative. This quantity is adopted for setting exclusion limits and a hypothesis is considered to be excluded at the $95 \%$ confidence level if the corresponding value of $\mathrm{CL}_{s}$ is less than 0.05. This method is also called "modified frequentist approach", since not the frequentistically determined confidence levels $\mathrm{CL}_{b}$ and $\mathrm{CL}_{s+b}$ but their ratio is used. 


\section{A.2 Expected and observed confidence levels}

The expected confidence levels are obtained by replacing the observed data configuration by a large number of simulated event configurations for the two hypotheses background only or signal+background. These can be used to estimate the expected sensitivity of a search and to compare the observed exclusion with the one expected with no signal present. The expected $\mathrm{CL}_{s}$ is then the median of the outcome of the large number of simulated experiments with background only event configuration.

Once the experimentally observed and the expected values of $\mathrm{CL}_{s}$ are derived as described above using the hypothesis of a Higgs production in the channel under study with SM strength (i.e. all BR and cross section scaling factors set to 1 ), the observed and expected values of $S_{95}$ can be numerically determined by varying the production rate of the channel by a scaling factor $s$ until

$$
\mathrm{CL}_{s}=0.05
$$

is reached. The scaling factor $s$ is then interpreted as observed or expected $S_{95}$, depending of whether the observed or expected confidence level $\mathrm{CL}_{s}$ has been used.

The numerical calculation at LEP has been done with tools described in [71] and [72], while the Tevatron results use different methods described in the individual references. 


\section{References}

[1] P. W. Higgs, Phys. Lett. 12 (1964) 132; Phys. Rev. Lett. 13 (1964) 508; Phys. Rev. 145 (1966) 1156;

F. Englert and R. Brout, Phys. Rev. Lett. 13 (1964) 321;

G. S. Guralnik, C. R. Hagen and T. W. B. Kibble, Phys. Rev. Lett. 13 (1964) 585 .

[2] S.L. Glashow, Nucl. Phys. B 22 (1961) 579;

S. Weinberg, Phys. Rev. Lett. 19 (1967) 19;

A. Salam, in: Proceedings of the 8th Nobel Symposium, Editor N. Svartholm, Stockholm, 1968.

[3] H. Nilles, Phys. Rept. 110 (1984) 1;

H. Haber and G. Kane, Phys. Rept. 117 (1985) 75;

R. Barbieri, Riv. Nuovo Cim. 11 (1988) 1.

[4] S. Weinberg, Phys. Rev. Lett. 37 (1976) 657;

J. Gunion, H. Haber, G. Kane and S. Dawson, The Higgs Hunter's Guide (Perseus Publishing, Cambridge, MA, 1990), and references therein.

[5] P. Fayet, Nucl. Phys. B 90 (1975) 104; Phys. Lett. B 64 (1976) 159; Phys. Lett. B 69 (1977) 489; Phys. Lett. B 84 (1979) 416;

H.P. Nilles, M. Srednicki and D. Wyler, Phys. Lett. B 120 (1983) 346;

J.M. Frere, D.R. Jones and S. Raby, Nucl. Phys. B 222 (1983) 11;

J.P. Derendinger and C.A. Savoy, Nucl. Phys. B 237 (1984) 307;

J. Ellis, J. Gunion, H. Haber, L. Roszkowski and F. Zwirner, Phys. Rev. D 39 (1989) 844;

M. Drees, Int. J. Mod. Phys. A 4 (1989) 3635;

U. Ellwanger, M. Rausch de Traubenberg and C.A. Savoy, Phys. Lett. B 315 (1993) 331 arXiv:hep-ph/9307322; Z. Phys. C 67 (1995) 665 arXiv:hep-ph/9502206; Nucl. Phys. B 492 (1997) 307 arXiv:hep-ph/9611251]; T. Elliott, S.F. King and P. White, Phys. Lett. B 351 (1995) 213 arXiv:hep-ph/9406303;

S.F. King and P. White, Phys. Rev. D 52 (1995) 4183 arXiv:hep-ph/9505326;

C. Panagiotakopoulos and K. Tamvakis, Phys. Lett. B 469 (1999) 145 arXiv:hep-ph/9908351;

C. Panagiotakopoulos and A. Pilaftsis, Phys. Rev. D 63 (2001) 055003 arXiv:hep-ph/0008268;

A. Dedes, C. Hugonie, S. Moretti and K. Tamvakis, Phys. Rev. D 63 (2001) 055009 arXiv:hep-ph/0009125.

[6] N. Arkani-Hamed, A. Cohen and H. Georgi, Phys. Lett. B 513 (2001) 232 arXiv:hep-ph/0105239;

N. Arkani-Hamed, A. Cohen, T. Gregoire and J. Wacker, JHEP 0208 (2002) 020 arXiv:hep-ph/0202089.

[7] N. Arkani-Hamed, S. Dimopoulos and G. Dvali, Phys. Lett. B 429 (1998) 263 arXiv:hep-ph/9803315]; Phys. Lett. B 436 (1998) 257 arXiv:hep-ph/9804398]; 
I. Antoniadis, Phys. Lett. B 246 (1990) 377;

J. Lykken, Phys. Rev. D 54 (1996) 3693 arXiv:hep-th/9603133;

I. Antoniadis and M. Quiros, Phys. Lett. B $392 \quad$ (1997) 61 arXiv:hep-th/9609209];

L. Randall and R. Sundrum, Phys. Rev. Lett. $\mathbf{8 3} \quad$ (1999) 3370 arXiv:hep-ph/9905221;

D. Cremades, L. E. Ibanez and F. Marchesano, Nucl. Phys. B 643 (2002) 93 arXiv:hep-th/0205074];

C. Kokorelis, Nucl. Phys. B 677 (2004) 115 arXiv:hep-th/0207234.

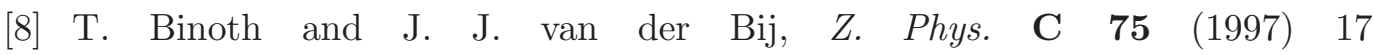
arXiv:hep-ph/9608245;

D. G. Cerdeno, A. Dedes and T. E. J. Underwood, JHEP 0609 (2006) 067 arXiv:hep-ph/0607157;

D. O'Connell, M. J. Ramsey-Musolf and M. B. Wise, Phys. Rev. D 75 (2007) 037701 arXiv:hep-ph/0611014.

[9] R. A. Porto and A. Zee, arXiv:0712.0448 [hep-ph].

[10] G. F. Giudice and O. Lebedev, Phys. Lett. B 665 (2008) 79 arXiv:0804.1753 [hep-ph]].

[11] R. Barate et al., Phys. Lett. B 565 (2003) 61 hep-ex/0306033.

[12] S. Schael et al., Eur. Phys. J. C 47 (2006) 547 hep-ex/0602042.

[13] [LEP Higgs Working Group for Higgs boson searches] arXiv: hep-ex/0107031; arXiv: hep-ex/0107032; arXiv: hep-ex/0107033 arXiv: hep-ex/0107034; arXiv: hep-ex/0107035.

[14] S. Heinemeyer, W. Hollik and G. Weiglein, Comput. Phys. Commun. 124 (2000) 76, arXiv:hep-ph/9812320; see: www.feynhiggs .de .

[15] S. Heinemeyer, W. Hollik and G. Weiglein, Eur. Phys. J. C 9 (1999) 343 arXiv:hep-ph/9812472.

[16] G. Degrassi, S. Heinemeyer, W. Hollik, P. Slavich and G. Weiglein, Eur. Phys. J. C 28 (2003) 133 arXiv:hep-ph/0212020.

[17] M. Frank, T. Hahn, S. Heinemeyer, W. Hollik, H. Rzehak and G. Weiglein, JHEP 0702 (2007) 047 arXiv:hep-ph/0611326.

[18] J. S. Lee, A. Pilaftsis, M. S. Carena, S. Y. Choi, M. Drees, J. R. Ellis and C. E. M. Wagner, Comput. Phys. Commun. 156 (2004) 283 [arXiv:hep-ph/0307377];

J. S. Lee, M. Carena, J. Ellis, A. Pilaftsis and C. E. M. Wagner, arXiv:0712.2360 [hep-ph].

[19] The ALEPH Collaboration, The DELPHI Collaboration, The L3 Collaboration, The OPAL Collaboration, The SLD Collaboration, The LEP Electroweak Working Group and The SLD Electroweak and Heavy Flavour Groups, Phys. Rept. 427 (2006) 257. arXiv:hep-ex/0509008. 
[20] J. Alcaraz et al. [ALEPH Collaboration and DELPHI Collaboration and L3 Collaboration and ], arXiv:hep-ex/0612034.

[21] The CDF Collaboration, CDF note 8954.

[22] The CDF Collaboration, CDF note 9284.

[23] The DØ Collaboration, Report FERMILAB-PUB-08-142-E, arXiv:0805.3556, submitted to Phys. Rev. Lett.

[24] The DØ Collaboration, Phys. Rev. Lett. 101 (2008) 071804, arXiv:0805.2491 [hep-ex].

[25] The CDF Collaboration, CDF note 9071.

[26] A. Djouadi, J. Kalinowski and M. Spira, Comput. Phys. Commun. 108 (1998) 56 arXiv:hep-ph/9704448.

[27] T. Hahn, S. Heinemeyer, F. Maltoni, G. Weiglein and S. Willenbrock, arXiv:hep-ph/0607308; U. Aglietti et al., arXiv:hep-ph/0612172;

See also: http://maltoni.web.cern.ch/maltoni/TeV4LHC/.

[28] O. Brein and W. Hollik, Phys. Rev. D 68 (2003) 095006 arXiv:hep-ph/0305321; Phys. Rev. D 76 (2007) 035002 [arXiv:0705.2744 [hep-ph]].

[29] T. Figy, C. Oleari and D. Zeppenfeld, Phys. Rev. D 68 (2003) 073005 arXiv:hep-ph/0306109.

[30] Alexander L. Read, private communication.

[31] LEP Higgs Working Group, unpublished.

[32] The CDF Collaboration, arXiv:0807.4493 [hep-ex].

[33] The CDF Collaboration, CDF note 9475.

[34] The DØ Collaboration, D0 note 5570.

[35] The DØ Collaboration, DØ note 5472.

[36] The CDF Collaboration, CDF note 9463.

[37] The DØ Collaboration, DØ note 5485.

[38] The CDF Collaboration, CDF note 7307.

[39] The DØ Collaboration, DØ note 5757.

[40] T. Aaltonen et al. [CDF Collaboration], arXiv:0809.3930 [hep-ex].

[41] The DØ Collaboration, Phys. Rev. Lett. 101 (2008) 051801 arXiv:0803.1514 [hep-ex]].

[42] The DØ Collaboration, DØ note 5737. 
[43] The DØ Collaboration, DØ note 5726.

[44] The CDF Collaboration, CDF note 9483.

[45] The DØ Collaboration, DØ note 5586.

[46] The CDF Collaboration, CDF note 9248.

[47] TEVNPH Working Group Collaboration for the CDF Collaboration and D0 Collaboration, Report FERMILAB-PUB-07-656-E, CDF note 8961, DØ note 5536, arXiv:0712.2383 [hep-ex].

[48] TEVNPH Working Group Collaboration for the CDF Collaboration and D0 Collaboration, Report FERMILAB-PUB-08-069-E, CDF note 9290, DØ note 5645, arXiv:0804.3423 [hep-ex].

[49] TEVNPH Working Group Collaboration for the CDF Collaboration and D0 Collaboration, Report FERMILAB-PUB-08-270-E, CDF note 9465, DØ note 5754, arXiv:0808.0534 [hep-ex].

[50] D. Graudenz, M. Spira and P. Zerwas, Phys. Rev. Lett. 70 (1993) 1372;

M. Spira, A. Djouadi, D. Graudenz and P. Zerwas, Nucl. Phys. B 453 (1995) 17 arXiv:hep-ph/9504378;

R. Harlander and W. Kilgore, Phys. Rev. Lett. 88 (2002) 201801 arXiv:hep-ph/0201206;

V. Ravindran, J. Smith and W. van Neerven, Nucl. Phys. B 665 (2003) 325 [arXiv:hep-ph/0302135];

C. Anastasiou and K. Melnikov, Nucl. Phys. B $646 \quad$ (2002) 220 arXiv:hep-ph/0207004;

S. Moch and A. Vogt, Phys. Lett. B 631 (2005) 48 [arXiv:hep-ph/0508265;

E. Laenen and L. Magnea, Phys. Lett. B $\mathbf{6 3 2}$ (2006) 270 arXiv:hep-ph/0508284.

[51] S. Catani, D. de Florian, M. Grazzini and P. Nason, JHEP 0307 (2003) 028 arXiv:hep-ph/0306211;

[52] R. V. Harlander and W. B. Kilgore, Phys. Rev. D 68 (2003) 013001 arXiv:hep-ph/0304035.

[53] A. Djouadi, Phys. Rept. 457 (2008) 1 arXiv:hep-ph/0503172.

[54] A. D. Martin, W. J. Stirling, R. S. Thorne and G. Watt, arXiv:0901.0002 [hep$\mathrm{ph}$.

[55] U. Langenegger, M. Spira, A. Starodumov and P. Trueb, JHEP 0606 (2006) 035 arXiv:hep-ph/0604156.

[56] O. Brein, A. Djouadi and R. Harlander, Phys. Lett. B 579 (2004) 149 arXiv:hep-ph/0307206;

M. L. Ciccolini, S. Dittmaier and M. Kramer, Phys. Rev. D 68 (2003) 073003 arXiv:hep-ph/0306234;

K. A. Assamagan et al. [Higgs Working Group Collaboration], arXiv:hep-ph/0406152. 
[57] T. Han, G. Valencia and S. Willenbrock, Phys. Rev. Lett. 69 (1992) 3274 arXiv:hep-ph/9206246;

J. M. Campbell and R. K. Ellis, Phys. Rev. D 60 (1999) 113006 arXiv:hep-ph/9905386;

T. Figy, C. Oleari and D. Zeppenfeld, Phys. Rev. D 68 (2003) 073005 arXiv:hep-ph/0306109;

E. Berger and J. Campbell, Phys. Rev. D $\mathbf{7 0}$ (2004) 073011 arXiv:hep-ph/0403194.

[58] C. Anastasiou, R. Boughezal and F. Petriello, arXiv:0811.3458 [hep-ph].

[59] U. Aglietti, R. Bonciani, G. Degrassi and A. Vicini, Phys. Lett. B 595 (2004) 432 arXiv:hep-ph/0404071, arXiv:hep-ph/0610033

S. Actis, G. Passarino, C. Sturm and S. Uccirati, Phys. Lett. B 670 (2008) 12 arXiv:0809.1301 [hep-ph]];

C. Anastasiou, S. Beerli, S. Bucherer, A. Daleo and Z. Kunszt, JHEP 0701 (2007) 082 arXiv:hep-ph/0611236].

[60] A. D. Martin, W. J. Stirling, R. S. Thorne and G. Watt, Phys. Lett. 652 (2007) 292 arXiv:0706.0459 [hep-ph]].

[61] P. H. Frampton, P. Q. Hung and M. Sher, Phys. Rept. 330 (2000) 263 arXiv:hep-ph/9903387.

[62] G. D. Kribs, T. Plehn, M. Spannowsky and T. M. P. Tait, Phys. Rev. D 76 (2007) 075016 arXiv:0706.3718 [hep-ph]].

[63] S. Heinemeyer, Int. J. Mod. Phys. A 212659 (2006) arXiv:hep-ph/0407244;

A. Djouadi, Phys. Rept. 459 (2008) 1. arXiv:hep-ph/0503173.

[64] T. Hahn, S. Heinemeyer, W. Hollik, H. Rzehak, G. Weiglein and K. Williams, Pramana 69 (2007) 861 arXiv:hep-ph/0611373.

[65] M. Carena, S. Heinemeyer, C. Wagner and G. Weiglein, Eur. Phys. J. C 26 (2003) 601 arXiv:hep-ph/0202167.

[66] Tevatron Electroweak Working Group and CDF Collaboration and D0 Collaboration, arXiv:0808.1089 [hep-ex].

[67] S. Heinemeyer, W. Hollik and G. Weiglein, JHEP 0006 (2000) 009 arXiv:hep-ph/9909540.

[68] M. S. Carena, J. R. Ellis, A. Pilaftsis and C. E. M. Wagner, Phys. Lett. B 495 (2000) 155 arXiv:hep-ph/0009212.

[69] M. S. Carena, J. R. Ellis, A. Pilaftsis and C. E. M. Wagner, Nucl. Phys. B 586 (2000) 92 arXiv:hep-ph/0003180.

[70] K. E. Williams and G. Weiglein, Phys. Lett. B 660 (2008) 217 arXiv:0710.5320 [hep-ph]].

[71] P. Bock, JHEP 0701 (2007) 080 arXiv:hep-ex/0405072].

[72] T. Junk, Nucl. Instrum. Meth. A 434 (1999) 435 arXiv:hep-ex/9902006. 